\title{
Numerical Simulation of Compressible Viscoelastic Liquids
}

\author{
I. J. Keshtiban, F. Belblidia and M. F. Webster* \\ Institute of Non-Newtonian Fluid Mechanics, \\ Department of Computer Science, \\ University of Wales, Swansea, SA2 8PP, UK.
}

\begin{abstract}
A stable and accurate time-marching pressure-correction/Taylor-Galerkin finite element algorithm is presented to accommodate low Mach number compressible and incompressible viscoelastic liquid flows. The algorithm is based on an operator splitting constructive process that discloses three fractional stages. For the compressible regime, a piecewise-constant density interpolation with gradient recovery is employed, for which the background theory and consistency of approach are discussed. The scheme is applied to contraction flows for Oldroyd model fluids, covering entry-exit flows and high pressure-drop situations. Stability and performance characteristics of the new algorithmic implementation are highlighted. Solutions are provided for a range of compressible settings, tending to the incompressible limit at vanishing Mach number.
\end{abstract}

KEY WORDS: Compressible liquid, viscoelastic, finite element, Taylor-Galerkin, pressure-correction, low Mach number, Oldroyd-B.

\footnotetext{
* Author for correspondence. Email: m.f.webster@swansea.ac.uk
} 


\section{Introduction}

In our previous studies [1,2], we presented a stable and accurate scheme to simulate weaklycompressible and incompressible viscous flows. There, the scheme was applied successfully to several benchmark test problems. In the compressible scenario, one observes spatial convergence-rates that reach a third-order for continuous problems. The algorithm performs well at low to vanishing Mach number $(M a)$, leading to the emergence of a unified scheme for both compressible and incompressible viscous flows. The aim of the present study is seen as a natural extension into the viscoelastic regime, to accommodate compressible and incompressible liquid flows, based upon the Oldroyd-B model fluid. We consider stability, accuracy and performance of the compressible viscoelastic algorithm for low to zero Mach number situations (approaching the incompressible limit). In addition, we conduct a parameter sensitivity analysis to assess convergence history for Weissenberg number (We) levels of order unity and for flows starting from quiescent initial conditions. We begin by providing some motivation for the present study.

Low Mach number flows play an important role, occurring widely in nature and in many industrial processes. In polymer processing operations, such as injection modelling and high-speed extrusion, pressure and flow rate may be large. Hence, compressibility effects within the viscoelastic regime may become important and influence resulting flow phenomena. From a physical perspective, the difference between incompressible and compressible flows lies in the propagation of longitudinal acoustic waves transmitted through the flow. For incompressible flows, the speed of such longitudinal waves approaches infinity, whereas the speed of transverse waves is finite. In contrast, for compressible flows, the speed of both waves takes on finite values. Moreover, this is manifest through a switch in equation type depending on the particular flow setting (incompressible to compressible). For example, within the unsteady viscous regime, the equations for compressible flow form a hyperbolic-parabolic system, whilst those for incompressible flow assume an elliptic-parabolic system. Nevertheless, it is preferable to work within a unified framework to accommodate algorithmically, in an automated fashion, with such type change. In the present study, this idea is pursued, based on local Mach number considerations, yet being capable of handling low Mach numbers, extending to the incompressible limit $(M a \approx 0)$.

Comprehensive literature reviews for viscoelastic incompressible flow may be found in Baaijens [3], Crochet et al. [4], Keunings [5] and Walters and Webster [6]. A variety of formulations have been developed over the last decade or so, such as finite volume (FV) methods [7,8], finite element method $[9,10,11]$, spectral collocation methods [12] and hybrid finite element-finite volume (FE/FV) methods $[13,14,15]$. The context of compressible viscous flow at low Mach number was covered extensively in our precursor study [1]. There, two principal numerical approaches were highlighted, being of densitybased and pressure-based construction. Apropos this context, numerical computation meets a significant challenge: transcending from low Mach numbers towards the zero Mach number, singular limiting state. The compressible viscoelastic domain is relatively unchartered in the literature. Georgiou [16] has addressed non-Newtonian inelastic (Carreau) fluid modeling for compressible flows, with particular interest in slip effects at the wall, relevant to time-dependent Poiseuille flow and extrudate-swell. Brujan [17] has derived an equation of motion for bubble dynamics, incorporating the effects of compressibility and viscoelastic proprieties for an Oldroyd-B model fluid. The objective there has been to analyse the physics of cavitation. In Barrett et al. [18], the equations of motion have been transformed into the Laplace domain to analyse a compressible dynamic viscoelastic hollow sphere problem.

From a numerical standpoint, the present formulation extends an incompressible fractional-staged time-stepping procedure to accommodate compressible viscoelastic flow regimes, for low to vanishing Mach number. This presents advance upon our earlier studies for viscoelastic incompressible flows [10], incorporating some recent developments adopted form the compressible viscous context $[1,2]$. The present formulation incorporates a semi-implicit Taylor-Galerkin/pressure-correction (TGPC) technique for mass and momentum, with a combination of two-step Lax-Wendroff and Crack-Nicolson time discretization procedures. This is a second-order pressure-correction scheme, solving for the temporal increment of pressure on the time-step. The discretization of stress is accomplished via a 
streamline-upwinding Taylor-Petrov-Galerkin technique, with velocity gradient recovery and hence, compatibility between stress and velocity gradient solution spaces. The equation of state for density represents the pressure dependence within the system, through the well-established modified form of the Tait model [19]. In the finite element representation, based upon our earlier observations and without loss of accuracy [1], density is interpolated in a piecewise-constant form (incompressible per element), with nodal recovery applied for density gradients.

At present, in the development of the algorithm, we restrict attention to isothermal flows. On a benchmark example of 4:1 planar contraction flow, we illustrate how the algorithm can meet the challenge of representing both compressible and incompressible liquid flows. We follow how algorithmic extension into the compressible regime has influenced the characteristics of the original incompressible implementation, particularly via convergence proprieties to steady-state and order of accuracy (in space and time [1]). The flow problem considered is that for an Oldroyd-B model, where it is acknowledged that a limiting Weissenberg number $\left(W e_{c r i t}\right)$ will be met.

The present article is organized as follows: the governing equations for compressible viscoelastic flow for Oldroyd-B model fluid are expounded in Section 2. In Section 3, we introduce the fractional equation stages of the Taylor-Galerkin/pressure-correction scheme, followed by the finite element (FE) spatial discretization adopted. In Section 4, we document solutions generated for the benchmark contraction flow test-problem. The schemes proposed are validated for consistency and accuracy via mesh refinement. Comparison, against incompressible counterparts and the literature, complements the high-order of accuracy achieved. This ranks above second-order for smooth flows. Careful attention is paid to temporal convergence histories for flows starting from quiescent initial conditions, attaining solutions at a unit level of Weissenberg number. Various strategies are highlighted to improve numerical convergence properties, specifically when dealing with the incompressible flow scenario.

\section{Compressible viscoelastic flow - governing equations}

The governing equations for compressible viscoelastic flow under isothermal conditions may be represented through conservation of mass and momentum, in conjunction with constitutive equation for stress and equation of state for density. The balance equations of mass and momentum ${ }^{\dagger}$ may be expressed in non-dimensional form, using standard differential operator notation and independent variables of time, $t$, and space, $x$ :

$$
\begin{aligned}
& \frac{\partial \rho}{\partial t}+\nabla \cdot(\rho U)=0 \\
& R_{e} \rho \frac{\partial U}{\partial t}=\left[\nabla .\left(2 \frac{\mu_{s}}{\mu} d+\tau\right)-R_{e} \rho U . \nabla U-\nabla P\right]
\end{aligned}
$$

where $U, \tau, P$ represent dependent variables of velocity, extra-stress and pressure, and $\nabla U$ the velocity gradient. Material properties are described through liquid density, $\rho$, relaxation time, $\lambda$ (see below), and viscosity, $\mu$. The total viscosity may be split into Newtonian (solvent), $\mu_{s}$, and polymeric (elastic), $\mu_{e}$, contributions, such that $\mu=\mu_{s}+\mu_{e}$. Non-dimensional parameters adopted are $\mu_{s}^{*}=\frac{\mu_{s}}{\mu}=\frac{1}{9}$ and $\mu_{e}^{*}=\frac{\mu_{e}}{\mu}=\frac{8}{9}$, and there is need to define the generalized rate-of-deformation tensor $d_{i j}=\frac{\left(\nabla U+(\nabla U)^{T}\right)}{2}-\frac{1}{3}(\nabla \cdot U) \delta_{i j}$.

We adopt the Oldroyd-B model fluid to characterise stress response, through the constitutive equation":

\footnotetext{
$\dagger$ Non-conservative form, see Hirsh [20]

\# Note, the recommended use of full-generalised form of $d$ in the UCM constitutive model by Oliveira et al. [8] for expediency in numerical convergence, even within the incompressible state.
} 
$W e \frac{\partial \tau}{\partial t}=-W e\left(U \cdot \nabla \tau-\tau \cdot \nabla U-(\tau \cdot \nabla U)^{T}\right)+2 \frac{\mu_{e}}{\mu} d-\tau$.

The dimensionless group numbers are those of Reynolds number and Weissenberg number, defined according to convention as,

$R_{e}=\frac{\rho^{0} U^{0} \ell^{0}}{\mu}, W e=\frac{\lambda U^{0}}{\ell^{0}}$.

Such dimensionless form is obtained by introducing characteristic scales on density $\rho^{0}$, viscosity $\mu$, length $\ell^{0}$ (exit half-channel width), velocity $U^{0}$ (exit flow, average velocity), and $\lambda$ the single relaxation time of the Oldroyd model-fluid. Note, for incompressible flow with constant density, the continuity equation reduces to: $\nabla \cdot U=0$.

To represent compressibility, we appeal to the modified Tait equation of state [19], to relate density to pressure, viz.,

$$
\frac{\tilde{P}+B}{\widetilde{P}_{0}+B}=\left(\frac{\rho}{\rho_{0}}\right)^{m} \quad \text { where augmented pressure, } \tilde{P}=P-\frac{1}{3} \operatorname{trace}\left(\tau+2 \frac{\mu_{s}}{\mu} d\right) \text {. }
$$

Here, power-index $m$ and constant $B$ are scalar parameters, and $\widetilde{P}_{0}, \rho_{0}$ denote reference scales for pressure and density, respectively. Note, in a strict sense, Eq.(5) is exact only for isentropic change. Although in the present analysis, the energy equation has been discarded ${ }^{\dagger}$, the Tait equation may be applied to reasonable precision more generally, since $m$ is independent of entropy, and $B$ and $\rho_{o}$ are constants [17]. This state law is often approximated to a linear form with index $m$ set to unity [21], an over-simplification which we may expose. After rearranging and differentiating the equation of state, we gather:

$$
\frac{\partial P}{\partial \rho}=m \rho^{m-1} \frac{\left(P_{0}+B\right)}{\rho_{0}^{m}}=\frac{m(P+B)}{\rho}=c_{(x, t)}^{2}
$$

where $c_{(x, t)}$ represents the speed of sound. By employing the chain rule upon Eq.(6) and taking difference operations over the time-step $\Delta t=\left(t_{n+1}-t_{n}\right)$, we may relate density increment to pressure increment at time instant $t_{n+1}$ through,

$$
\frac{\Delta \rho^{n+1}}{\Delta t}=\frac{1}{c_{(x, t)}^{2}} \frac{\Delta P^{n+1}}{\Delta t} \text {. }
$$

We have recourse to such a relationship below in the derivation of the particular fractional-stage equations, to realise a compressible temporal evolutionary expression for pressure (see reference [1] for more details).

\section{Taylor-Galerkin/pressure-correction scheme and FE discretisation}

The general framework of the Taylor-Galerkin/pressure-correction (TGPC) scheme is based on a time-stepping procedure, involving two distinct phases within each time step. The first phase involves a Taylor-Galerkin scheme, expounded through a two-step, Lax-Wendroff time stepping procedure. This represents a predictor-corrector doublet for velocity and stress, which initially calculates predicted fields $(U, \tau)^{n+1 / 2}$, prior to computing a non-divergence-free velocity field $U^{*}$ and an updated stress field $\tau^{n+1}$. The second phase is a pressure-correction scheme that ensures second-order accuracy in time. This generates an equation for temporal pressure-difference and a Poisson equation in incompressible flow. A third and final phase is a correction stage that recaptures the velocity field

\footnotetext{
$\dagger$ Here, we are interested in low Reynolds number isothermal flows, where kinetic considerations are negligible.
} 
$U^{n+1}$ at the end-of-time step loop. By implementing a semi-implicit Crack-Nicolson treatment for diffusion terms, a semi-discrete incremental form of the TGPC scheme may be derived, devoid of heavy stability restriction imposed through diffusion operators. This scheme may be presented in a three-fractional stage form, viz.,

Stage-1a:

$$
\begin{aligned}
& 2 R_{e} \rho \frac{U^{n+\frac{1}{2}}-U^{n}}{\Delta t}=\left[\nabla \cdot\left(2 \mu_{s} D+\tau\right)-R_{e} \rho U \cdot \nabla U\right]^{n}-\nabla P^{n}+\nabla \cdot \mu_{s}\left(D^{n+\frac{1}{2}}-D^{n}\right) \\
& 2 W_{e} \frac{\tau^{n+\frac{1}{2}}-\tau^{n}}{\Delta t}=\left[2 \mu_{e} D-\tau-W_{e}\left\{U \cdot \nabla \tau-\tau \cdot \nabla U-(\tau \cdot \nabla U)^{T}\right\}\right]^{n}
\end{aligned}
$$

Stage-1b:

$$
\begin{aligned}
& R_{e} \rho \frac{U^{*}-U^{n}}{\Delta t}=\left[\nabla \cdot \tau-R_{e} \rho U \cdot \nabla U\right]^{n+\frac{1}{2}}-\left[\nabla \cdot\left(2 \mu_{s} D\right)-\nabla P\right]^{n}+\nabla \cdot \mu_{s}\left(D^{*}-D^{n}\right) \\
& W_{e} \frac{\tau^{n+1}-\tau^{n+\frac{1}{2}}}{\Delta t}=\left[2 \mu_{e} D-\tau-W_{e}\left\{U \cdot \nabla \tau-\tau \cdot \nabla U-(\tau \cdot \nabla U)^{T}\right\}\right]^{n+\frac{1}{2}}
\end{aligned}
$$

Stage-2:

$$
\frac{1}{c_{(x, t)}^{2}} \frac{P^{n+1}-P^{n}}{\Delta t}-\Delta t \theta \nabla^{2}\left(P^{n+1}-P^{n}\right)=-\left[\rho^{n} \nabla \cdot U^{*}+\nabla \rho^{n} . U^{*}\right]
$$

Stage-3:

$$
R_{e} \rho \frac{U^{n+1}-U^{*}}{\Delta t}=-\theta \nabla\left(P^{n+1}-P^{n}\right)
$$

Note, the momentum equations for compressible and incompressible flows are practically identical, bar variation in density. Differences emerge due to the various alternative forms of the continuity equation. Hence, Eq.(12) displays some notable features (see [1,2] for detailed derivation): the first term on the left-hand-side is a first-order time derivative representation, whilst the second term is governed by a Laplacian operator (elliptic properties). In addition, on the right-hand-side, density is a direct function of pressure, that is to be interpreted via the Tait equation of state. When the flow is incompressible, the speed of sound asymptotes to infinity. In this context, the first term on the lefthand-side vanishes along with the second term on the right-hand-side, and elliptic character dominates Eq.(12). Alternatively, switching to compressible flows, the balance of equation-type-domination adjusts between elliptic and hyperbolic, the extent of type-mix depending upon the Mach number level (local compressibility).

A Galerkin finite element spatial discretization is adopted, based on triangular elements in two dimensions. This employs a piecewise continuous quadratic interpolation $\phi(x)$ for velocity and stress, and linear interpolation $\psi(x)$ for pressure, of the form:

$U(x, t)=U_{j}(t) \phi_{j}(x), \tau(x, t)=T_{j}(t) \phi_{j}(x), \mathrm{j}=1,6$ and $P(x, t)=P_{k}(t) \psi_{k}(x), \quad \mathrm{k}=1,3$.

Time-dependent velocity, stress and pressure nodal-vectors are represented as $U, T$ and $P$, respectively. For density, piecewise-constant interpolation over an element is considered, which necessitates recovery to gather density gradient representation. Algebraic solvers for such systems are dealt with extensively elsewhere [22,23]. Briefly, a direct solution method is employed at the pressure equation stage, whilst an efficient element-by-element Jacobi iteration is invoked over the remaining stages. 
As alluded to above, stability of the scheme is enhanced by appealing to a semi-implicit version of the scheme. The discretized equation for compressible viscoelastic flow may be expressed in fullydiscrete matrix form, via TGPC-stages1-3, viz.

Stage-1a:

$\left[2 R_{e} \frac{M_{\rho}}{\Delta t}+\frac{\mu_{s}}{2} S_{U}\right]\left(U^{n+\frac{1}{2}}-U^{n}\right)=\left\{-\left[\mu_{s} S_{U}+R_{e} N_{\rho}(U)\right] U-B T\right\}^{n}+L^{T} P^{n}$
$2 W_{e} \frac{M}{\Delta t}\left(T^{n+\frac{1}{2}}-T^{n}\right)=\left[2 \mu_{e} M\left(L+L^{T}\right)-\left\{M+W_{e} N(U)\right\} T+W_{e}\left\{N_{e}(T) L+\left(N_{e}(T) L\right)^{T}\right\}\right]^{n}$

Stage-1b:

$\left[R_{e} \frac{M_{\rho}}{\Delta t}+\frac{\mu_{s}}{2} S_{U}\right]\left(U^{*}-U^{n}\right)=\left\{-\left[\mu_{s} S_{U}+R_{e} N_{\rho}(U)\right] U-B T\right\}^{n+\frac{1}{2}}+L^{T} P^{n}$

$W_{e} \frac{M}{\Delta t}\left(T^{n+1}-T^{n}\right)=\left[2 \mu_{e} M\left(L+L^{T}\right)-\left\{M+W_{e} N(U)\right\} T+W_{e}\left\{N_{e}(T) L+\left(N_{e}(T) L\right)^{T}\right\}\right]^{n+\frac{1}{2}}$

Stage-2:

$\left[\frac{M_{C}}{\Delta t}+\theta \Delta t K\right]\left(P^{n+1}-P^{n}\right)=-L^{\rho} U^{*}$

Stage-3:

$R_{e} \frac{M_{\rho}}{\Delta t}\left(U^{n+1}-U^{*}\right)=\theta L^{T}\left(P^{n+1}-P^{n}\right)$

where

$$
\begin{array}{lll}
\left(M_{\rho}\right)_{i j}=\int_{\Omega} \rho \phi_{i} \phi_{j} d \Omega, & (M)_{i j}=\int_{\Omega} \phi_{i} \phi_{j} d \Omega, & \left(\mathrm{M}_{C}\right)_{i j}=\int_{\Omega} \frac{\psi_{i} \psi_{j}}{c_{(x, t)}^{2}} d \Omega \\
\left(N_{\rho}\right)_{i j}=\int_{\Omega} \rho \phi_{i}\left(U \cdot \nabla \phi_{j}\right) d \Omega, & (N)_{i j}=\int_{\Omega} \phi_{i}\left(U \cdot \nabla \phi_{j}\right) d \Omega, & \left(N_{e}\right)_{i j}=\int_{\Omega} \phi_{i}\left(T \cdot \nabla \phi_{j}\right) d \Omega \\
(B)_{i j}=\int_{\Omega} \phi_{i} \nabla \phi_{j} d \Omega, & (K)_{i j}=\int_{\Omega} \nabla \psi_{i} \cdot \nabla \psi_{j} d \Omega \\
\left(L_{k}\right)_{i j}=\int_{\Omega} \psi_{i}\left(\nabla \cdot \phi_{j}\right)_{k} d \Omega, & \left(L_{k}^{\rho}\right)_{i j}=\int_{\Omega} \psi_{i}\left\{\psi_{l} \rho_{l} \frac{\partial \phi_{j}}{\partial x_{k}}+\frac{\partial \psi_{l}}{\partial x_{k}} \rho_{l} \phi_{j}\right\} d \Omega \\
\left(S_{U}\right)_{i j}=\left(S_{l m}\right)_{i j}, l, m=1,2 & \left(S_{11}\right)_{i j}=\int_{\Omega} \mu\left(2 \frac{\partial \phi_{i}}{\partial x} \frac{\partial \phi_{j}}{\partial x}+\frac{\partial \phi_{i}}{\partial y} \frac{\partial \phi_{j}}{\partial y}-\frac{2}{3} \frac{\partial \phi_{i}}{\partial x} \frac{\partial \phi_{j}}{\partial x}\right) d \Omega \\
\left(S_{12}\right)_{i j}=\int_{\Omega} \mu \frac{\partial \phi_{i}}{\partial y} \frac{\partial \phi_{j}}{\partial x} d \Omega, & \left(S_{22}\right)_{i j}=\int_{\Omega} \mu\left(\frac{\partial \phi_{i}}{\partial x} \frac{\partial \phi_{j}}{\partial x}+2 \frac{\partial \phi_{i}}{\partial y} \frac{\partial \phi_{j}}{\partial y} \frac{2}{3} \frac{\partial \phi_{i}}{\partial y} \frac{\partial \phi_{j}}{\partial y}\right) d \Omega
\end{array}
$$

From a computational standpoint, the main modification introduced to the incompressible TGPC scheme (see reference [10]) to handle the compressible regime has arisen at stage-2. This is achieved by the introduction of the two new matrices $M_{C}$ and $L^{\rho}$, governed by compressibility considerations. In addition, in the definition of the diffusion matrix $S_{U}$, an additional term is appended $\left(-\frac{2}{3} \frac{\partial \phi_{i}}{\partial x} \frac{\partial \phi_{j}}{\partial x}\right)$ within $S_{11,}$ and likewise, $\left(-\frac{2}{3} \frac{\partial \phi_{i}}{\partial y} \frac{\partial \phi_{j}}{\partial y}\right)$ in $S_{22}$. With piecewise-constant density 
interpolation over an element, some matrix simplification ${ }^{\dagger}$ arises: $\left(M_{\rho}\right)_{i j}=\sum_{\Omega_{e}} \rho_{e}\left(M^{e}\right)_{i j}$ and $\left(N_{\rho}\right)_{i j}=\sum_{\Omega_{e}} \rho_{e}\left(N^{e}\right)_{i j}$, where $\left(M^{e}\right)_{i j}=\int_{\Omega_{e}} \phi_{i} \phi_{j} d \Omega_{e}$ and $\left(N^{e}\right)_{i j}=\int_{\Omega_{e}} \phi_{i}\left(U \cdot \nabla \phi_{j}\right) d \Omega_{e}$. We note that the incompressible second stage of the TGPC scheme collapses to:

$[\theta \Delta t K]\left(P^{n+1}-P^{n}\right)=-L U^{*}$.

This completes our discourse on scheme derivation and discretization issues.

\section{Scheme implementation and numerical solutions}

The benchmark problem of flow through an abrupt 4:1 contraction for an Oldroyd-B fluid is recognised as a formidable test problem, in terms of stability at high Weissenberg number. It is welldocumented in the literature on viscoelastic incompressible flow (for example, see references: [6,24]). Here, it is chosen to validate our asymptotic 'zero' Mach number solutions against published incompressible results. In addition, consistency, accuracy and behaviour of the more compressible solutions is monitored for high levels of Weissenberg number. For this problem, the total length of the planar channel is 76.5 units (see Figure 1). No-slip boundary conditions are assumed on solid boundaries. At the inlet, transient boundary conditions (bc) are imposed reflecting build-up through flow-rate (Waters and King, (W\&K) [25]), generating set profiles for normal velocity $\left(U_{x}\right)$ and stress $\left(\tau_{\mathrm{xx}}, \tau_{\mathrm{xy}}\right)$, displaying vanishing cross-sectional components in velocity $\left(\mathrm{U}_{\mathrm{y}}\right)$ and stress $\left(\tau_{\mathrm{yy}}\right)$. In contrast, at the exit, weak-form natural boundary conditions are established, via boundary integrals (B.I.) and momentum equation representation, where once more, $U_{y}$ vanishes. A pressure reference level is set to zero at the outlet. We take advantage of flow symmetry about the horizontal central axis running through the domain, computing solutions on half the problem domain. Reynolds number is set to unity allowing some inertia to enter the problem. To accommodate different flow regimes, our strategy has been to alter the values of the Tait parameter-set $(m, B)$. These have been adjusted between those representing low compressible flow conditions, say $\left(4,10^{2}\right)$, to a highly incompressible state, typically $\left(10^{4}, 10^{4}\right)$. Then, corresponding solutions may be compared unequivocally to those for incompressible prevailing assumptions.

\subsection{Trends in temporal convergence history}

The unified framework may be assessed with respect to time-stepping convergence history tolerance to steady-state and spatial accuracy properties. To this end, three different meshes are employed, M1, M2 and M3, covering increasing levels of refinement within the contraction zone. Triangular element mesh structure is illustrated in Figure 2 and mesh characteristics are quantified in Table I, where details are recorded for total numbers of elements, nodes, degrees of freedom, corner mesh density and minimum and maximum element size (see also, Baloch et al. [9] and Matallah et al. [10]). Here, a multi-block meshing strategy is employed, with conformal mapping in each sub-block and matching of boundary nodes at interfaces. To access the time-stepping convergence history a temporal relative-increment $L_{2}$-norm is defined to govern time-stepping convergence history against a set tolerance, Tol $_{t}$, viz.:

$\dagger\left(M_{\rho}\right)_{i j}=\int_{\Omega} \rho \phi_{i} \phi_{j} d \Omega=\sum_{\Omega_{e}}\left(\int_{\Omega_{e}} \rho \phi_{i} \phi_{j} d \Omega_{e}\right)=\sum_{\Omega_{e}}\left(\rho_{e} \int_{\Omega_{e}} \phi_{i} \phi_{j} d \Omega_{e}\right)=\sum_{\Omega_{e}} \rho_{e}\left(M_{i j}^{e}\right)$ 


$$
E^{T}(X)=\frac{\left\|X^{n+1}-X^{n}\right\|}{1+\left\|X^{n+1}\right\|} \leq \text { Tol }_{t} .
$$

a- Numerical parameter sensitivity analysis (compressible flow)

First, within the compressible context, we conduct a numerical parameter sensitivity analysis on time-step $(\Delta t)$ and mass iteration number $\left(m_{i t n}\right)$, setting the Tait parameter pairing to $(m, B)=\left(4,10^{2}\right)$. Here, computational testing is based on mesh M1, with Weissenberg number set to unity and commencing from initial conditions (ic) of a quiescent state. History tolerance results governing pressure $\left(E^{\mathrm{T}}(\mathrm{p})\right)$, velocity $\left(\mathrm{E}^{\mathrm{T}}(\mathrm{U})\right)$ and stress $\left(\mathrm{E}^{\mathrm{T}}(\mathrm{T})\right)$ are displayed in Figure 3. The relevant parameter values are those of: $\Delta t=10^{-2}, 10^{-3}, 10^{-4}$; and $m_{i t n}=1,3$ and 5. For the compressible flow setting, results demonstrate that history convergence norm increments are relatively insensitive to adjustment in timestep beyond $10^{-3}$ and mass iteration number greater than 3 . As stability is maintained, it is apparent that by increasing time-step value (interpreted as a fraction of the local Courant number), less time-steps and effort are demanded to achieve a specified relative tolerance level $\left(10^{-7}\right)$ equating to steady-state. An important observation concerns the start-up phase. There, some oscillation is apparent in history tolerance, as anticipated for Waters and King kinematic start-up conditions (representative for straight channels). These oscillations are damped away rapidly, whilst the flow evolves to a steady-state, independent of the parameter settings employed.

\section{b- Incremental continuation in Weissenberg number (incompressible flow)}

Next, we turn attention to incompressible viscoelastic computations. A well-recognised and successful strategy to reach a stable steady-state at larger Weissenberg number levels (current target, $W e=1.0$ ) is to employ incremental continuation in Weissenberg number (order zero continuation). This procedure is illustrated through sample results in Figure 4 with numerical parameters set throughout of $\left(\Delta t=10^{-3}, m_{i t n}=3\right.$, mesh M2). In this manner, and commencing at the outset from a quiescent state we first gather a solution at $W e=0.1$. Subsequently, the $W e=0.1$ steady-state solution is taken up as the initial starting phase, to derive a solution for $W e=1.0$. For models such as Oldroyd, a limiting level of Weissenberg number $\left(W e_{\text {crit }}\right.$ ) is commonly encountered in complex flows (see $\left.[3,13,26]\right)$. Here, we restrict ourselves to validation at attainable solution levels of unity, to draw out and contrast algorithmic properties of the various alternative implementations. Even at the level of $W e=1.0$, attempting a direct solution procedure (mimicking true time evolution) and starting from a quiescent state, reveals a persistent periodic non-convergence pattern in convergence history, as demonstrated in Figure $4 \mathrm{~b}$. This is a common oscillatory feature at peak $\mathrm{We}$-levels with many stable algorithms, where monotonic convergence lies on a stability threshold, dependent upon the size of initial perturbation placed upon the system (given fixed discrete parameters of mesh size and time-step). In like manner, divergence at larger $\mathrm{We}$-values would ensue.

\section{c- Under-relaxation procedure (incompressible flow)}

Numerical instability, exposed through temporal convergence history tolerances, may be attributed to spatial or temporal discretization error [12]. Here, stabilization methods, adequate for steady equation systems, may prove ineffective for unsteady systems. Moreover, approximation error accumulated through the equation splitting during the fractional-staged procedure, may itself be a source of instability. Fietier et al. [12] have investigated numerical stability and presented timedependent algorithms for viscoelastic flows, employing spectral element methods. In their study, some stabilization strategies are proposed for flow in simple geometrical configurations. One such proposal is a filtering technique, applied after each time step in an element-by-element fashion on both velocity and stress fields. Still further suggestions for stabilization procedures may be found elsewhere $[3,10]$.

Through the current implementation, numerical stability can be sustained to larger We-levels, by appealing to solution under-relaxation. This is demonstrated starkly in Figure 4c, where solution underrelaxation is applied equally to all variables $\left\{r_{U}, r_{\tau}, r_{P}\right\}$, at the end of a complete time-step loop. History convergence tolerance oscillations are dramatically removed and monotonic convergence is essentially recovered. We observe that such a relaxation ploy may be interjected on each fractional-stage equation 
within the time-step loop, so that, solution relaxation may be interpreted via time-step scaling upon each equation-stage time-step (local time-step, per equation). The reader is referred to Appendix for more details. Hence, one arrives at the justification for adjustable internal time-steps [27], to be judged as acting on each fractional-stage solution variable and indeed, expressing dependency upon the mesh selected (fraction of the Courant number).

For viscoelastic incompressible counterparts, we consider the consequences of under-relaxation in some greater detail to gather optimality. To this end, again we adopt mesh M2, and target $W e=1.0$ solutions from quiescent initial conditions. Figure 5a presents the convergence history scenario without relaxation, for ease of comparison. End-of-3-stages relaxation factors $\left(r_{i}\right.$, per variable $\left.i\right)$ are applied to the solution field $\chi$ of the form:

$\chi_{\text {relax }}^{n+1}=r_{i} \chi^{n+1}+\left(1-r_{i}\right) \chi^{n}$.

Convergence histories for constant parameter-setting with $\left\{r_{U}, r_{\tau}, r_{P}\right\}=\{0.3,0.3,0.3\}$ are provided in Figure $5 \mathrm{~b}$. This choice is insufficient to damp the persistent oscillatory pattern that has emerged. An optimal level is observed at $\{0.7,0.7,0.7\}$ in Figure 5c, which has the desired suppression and smoothing effect over the prior oscillatory pattern. One may isolate the influence of relaxation to each individual variable (and stage thereby). So, for example, the setting of $\{0.7,0.7,0.0\}$ of Figure $5 \mathrm{~d}$ reveals insensitivity according to $r_{P}$ (hence, on $\Delta t_{P}$ scaling). As demonstrated in Appendix, underrelaxing the local time-step (on velocity) at stage-1 is conveyed to stage-2 local time-step, even without under-relaxing the pressure variable. In contrast, removing velocity under-relaxation, with $\{0.0,0.7,0.0\}$-choice, demonstrates in Figure 5e, the crucial role of this factor alone: optimal $r_{U}=0.7$. Removing stress under-relaxation with $\{0.7,0.0,0.0\}$-setting (as in Figure 5f) accelerates temporal convergence rates by fifty percent, achieving the tolerance target in half the time. One concludes, that primarily, only velocity relaxation should be applied. Finally, applying velocity under-relaxation with optimal factors for mesh M2: $\{0.7,0.0,0.0\}$ at end-of-first-stage or at end-of-third-stage (end-ofcomplete-stage cycle) delivers identical history tolerance behaviour, as demonstrated in Figure 6. This confirms the crucial impact and importance of under-relaxation upon stage-1 equations, prior to the remaining fractional-stages within the time-step loop.

\section{d-Under-relaxation: incompressible, weakly-compressible, compressible}

Based upon the above remarks, we illustrate, in Figure 7, the different history tolerance results across the series of three meshes employed for incompressible, weakly-compressible $\left((m, B)=\left(10^{4}, 10^{4}\right)\right)$ and compressible $\left((m, B)=\left(4,10^{2}\right)\right)$ flows. For all scenarios (fluid and mesh) a $W e=1.0$ is targeted from a quiescent initial state. The first observation is that, independent of mesh employed or flow type, convergence history tolerance always commences with an oscillatory pattern, typical of transient startup conditions. About ten to fifteen time units are necessary to dampen these oscillations and recover a smooth convergence pattern. For incompressible and weakly-compressible flows, based on mesh M2 and $\mathrm{M} 3$, under-relaxation is necessary to reach the specified tolerance level. As anticipated by reducing mesh size from M2 to M3, optimum levels of the relaxation factors imply lesser need for relaxation. Note that for these flows, a time-step of $10^{-3}$ is applied, leading to steady-state solutions within twenty to fifty time units. Here, we point out that the choice of time-step is not only governed by the Courant number restriction, but also by the size of Weissenberg number. For compressible flow conditions, relaxation is unnecessary at this level of Weissenberg number, to reach the specific tolerance and enhanced smoothness of convergence is apparent upon the three test meshes. This is due mainly to improved equation conditioning at stage-2, through the modifications to accommodate for compressibility considerations (see Eq.(19), $M_{c}$ and $\left.L^{\rho}\right)$. Here, a larger time-step is permitted $\left(\Delta t=10^{-2}\right)$. Independent of the spatial discretization employed, a tight window of temporal convergence is observed, displaying a uniform and smooth trend. For compressible flow, independent of meshing, less time-steps and CPU-time is demanded to reach a steady-state solution when compared to counterpart incompressible flow settings. Finally, mesh refinement does not necessarily demand stringent reduction in time-step to reach the desired tolerance level (as is the case in the incompressible context). This bodes well for numerical continuation between the different levels of compressibility. 


\subsection{Mesh refinement}

Convergence of solutions in all component variables has been confirmed through mesh refinement on the series of meshes employed. Finally, consistency of the scheme is highlighted by the ability of the scheme to accommodate weakly-compressible flow scenarios. There similar results are derived for incompressible and weakly-compressible representations.

Having established satisfactory convergence trends, independent of flow type or mesh employed, we turn to interrogate field solution quality through pressure, principal stress $\mathrm{N}_{1}$ (first normal stressdifference) and shear stress contour plots. All our results thus far have been for $W e=1.0$ and $R e=1.0$. Each variable figure represents nine contour plots. Incompressible flow results are displayed on the left, weakly-compressible in the middle and compressible to the right. Solutions based on mesh M1 are illustrated at figure-top, M2 at centre, and M3 over figure-bottom. In this manner and for each variable, it is practical to contrast scheme accuracy, based on mesh refinement, and scheme consistency whilst increasing Mach number (from the incompressible toward the compressible flow regime). In Figure 8, around the contraction zone, similar pressure contour patterns at equitable levels are observed for incompressible and weakly-compressible representations. Pressure-drop increases with mesh-density in all instances, being slightly higher for weakly-compressible as compared to incompressible flow setting. For the more compressible regime, it is apparent that pressure-drop is higher (about ten percent) than with other flow settings, relating directly to compressibility effects. Similar conclusions may be drawn on principal stress contour plots presented in Figure 9. Here, the maximum level of stress is considerably larger upon mesh M3, when compared with M2 or M1, independent of flow representation. This increase is about ninety to ninety-five percent, indicating the strong presence of a singularity located at the re-entrant corner. Field distributions show little disparity between flow settings upon the remainder of the domain, away from the downstream-wall, increasing thereupon. Shear stress contour plots are presented in Figure 10. Here again, there is a doubling in maximum stress level between mesh M1 and M3 solutions, indicating again the significant presence of a singularity. Note, that for each variable, the contour plots display similar patterns through mesh refinement or flow-type under consideration. Incompressible stress fields are corroborated by their close agreement with those in the published literature (see $[13,14])$.

Based on the finest mesh M3, streamlines contours plots for incompressible and compressible flows are displayed in Figure 11. Similar incompressible streamlines contours at $W e=1.0$ are presented in reference [14]. Contour distributions show little disparity between flow settings, except at the centre of the vortex zone, where the stream function peaks $\left(* 10^{-4}\right)$ increase from -1.86 (compressible) to -2.06 (incompressible), and stream function maxima increase from 1.00 (incompressible) to 1.28 (compressible). Finally, for compressible flow based on mesh M3, density and Mach number contours are provided in Figure 12. Note, density contours are non-parallel in the upstream or downstream channel sections (as with pressure). This is due to the fact that under viscoelastic consideration, density is a function of the augmented pressure $\widetilde{P}$, which takes into account the trace of stress (see Tait equation Eq.(5)). An increase of $28 \%$ in density arises between the inlet and outlet. Outlet Mach numbers of $M a=0.093$ are approached under such compressible flow conditions.

\subsection{Stress boundary layer development}

To highlight the re-entrant corner solution for $W e=1.0$ solutions, stress profiles $\left(\tau_{x x}, \tau_{x y}, \tau_{y y}\right)$ at $y=0.3$ units (see Figure 1) are illustrated in Figure 13, across the three meshes and flow scenarios. For clarity in plotting, a shift in the position of the re-entrant corner for the different meshes has been applied. As anticipated there are no noticeable differences in stress plot results for incompressible and weaklycompressible representations. All plots reflect a prominent stress peak at the re-entry corner. The level of the peak increases with mesh refinement, and also, with greater levels of compressibility.

For the more compressible flow, a major feature lies in the growth of stress, $\tau_{\mathrm{xx}}$, in the boundary layer along the downstream wall (monotonic growth at an angle $8.5^{\circ}$ ). A corresponding feature arises to a lesser degree in the shear stress component, $\tau_{\mathrm{xy}}$. Such an expansion/growth of boundary layer is non-existent in incompressible or weakly-compressible flow settings. Finally, note that compressibility does not mitigate the strength of the corner solution singularity in stress. 
Careful analysis of the boundary layer development is conducted upon the principal first normal stress-difference, $\mathrm{N}_{1}$. To this end, variation in stress across the channel, cross-section $y=3$ to $y=4$ units, is plotted at different downstream positions (see Figure 14). Results are presented in Figure 15 based on mesh M3 for the three flow representations. From a downstream position (here, $x=30$ units) there is barely any discernible variation in stress field through the channel cross-section for the incompressible or weakly-compressible flows. In contrast, there is more widening and gradual growth of the boundary layer along the wall for the more compressible flow conditions. As illustrated in Figure 16, near the contraction zone ( $22.2 \leq x \leq 30$ units, contraction at $x=22$ units), we observe boundary layer growth for all flows with increasing x-position along the downstream wall. The boundary layer width for incompressible and weakly-compressible flow reaches a maximum rapidly (here, at $x=30$ units), after which no further expansion is encountered. In Figure 17, where for plotting clarity the cross-section dimension has been zoomed some forty times, three-stress levels (contour values, 1, 5 and 15 for $\tau_{\mathrm{xx}}$, and $0.5,1.8$ and 2.6 for $\tau_{\mathrm{xy}}$ ) are plotted along the downstream channel wall. This demonstrates the monotonic growth in the stress boundary layer for compressible flow; in contrast, no growth is apparent for either of the incompressible or weakly-compressible flow instances. As an overall remark, the main disparity between incompressible and compressible flow representations is related to the levels of stress peak at the re-entry corner and the monotonic stress growth along the boundary layer, throughout the downstream wall. In the incompressible state, such growth manifests a constant (limiting) level and width, established fairly rapidly beyond the corner after the stress boundary layer has formed.

\section{Conclusions}

This study has provided new insight into the finite element modelling of viscoelastic compressible flows, where we have consciously focused on the Oldroyd model, the Tait equation of state, and planar contraction flows. We have proposed a fresh look at pressure-correction type formulations to tackle such a setting, so utilising pressure as a primary variable emanating from conventional incompressible flow representation. In this manner, we have accurately and consistently resolved both evolutionary and steady-state flows, covering the low Mach number regime. A unified (single) finite element scheme is advocated, with flexibility to cover compressible, weakly-compressible, and incompressible flow situations. Various aspects of study have revealed: (i) the role and importance of relaxation across fractional-staged equations; (ii) sensitivity of the scheme to selection of $\left\{\Delta t, h, m_{i t n}\right\}$-parameters, within the different flow settings; (iii) convergence trends and contrast of flow field response.

A major observation is the heavy-side influence that compressible equation structure has on temporal, monotonic, in-phase convergence properties. This extends equally to the asymptotic limit and weakly-compressible scenario, that mimics (slightly) incompressible flow $\left(M a \simeq 10^{-4}\right)$. Hence, the weakly-compressible assumption may be usefully employed to numerically improve convergence properties for incompressible viscoelastic solutions.

Pressure drops rise when greater levels of compressibility are incorporated (here by order 10\%). We observe the fact that solution singularity (stress at re-entrant corner) may actually increase in a more compressible setting, whilst downstream-wall stress boundary layers broaden along the wall, away from initial inception, just beyond the re-entrant corner. This is in stark contrast to the incompressible scenario, where such boundary layer structure sustains its initial width far downstream of its initial onset. Hence, stress boundary layers are provoked almost directly beyond the re-entrant corner in all flows studied, yet it is the tight width of the incompressible boundary layer that emerges as the single most severe physical phenomena to resolve. Extensions to the current study shall be oriented towards seeking solutions up to critical levels of Weissenberg number, and considering temporal adjustment of the compressible parameter set.

\section{Acknowledgements}

The financial support of EPSRC grant GR/R46885/01 is gratefully acknowledged. 


\section{Appendix: Fractional staged equations and relaxation procedure}

The relaxation procedure may be applied at different TGPC stages and has a direct effect on the local time-step-stage value. To clarify this point, let us consider the first and second stages of TGPC scheme for incompressible fluid in compact matrix form:

$$
\begin{aligned}
& A_{U}\left(U^{*}-U^{n}\right)=\Delta t b_{U} \\
& K\left(P^{n+1}-P^{n}\right)=\frac{1}{\theta \Delta t} L U^{*} .
\end{aligned}
$$

Here, $n$ denotes the time level, $\Delta t$ the local time-step, $U^{*}, U, P$, nodal vectors of non-divergence-free velocity, velocity and pressure, respectively, $b_{U}$ the right-hand-side of stage- $1 \mathrm{~b} . A_{U}$ and $K$ are velocity and pressure governing matrices (mass and stiffness).

In order to relax velocity by $\alpha_{U}$ at the end of stage-1, we introduce a relaxed non-divergence-free velocity $\tilde{U}^{*}$, viz.:

$\tilde{U}^{*}=\alpha_{u} U^{*}+\left(1-\alpha_{u}\right) U^{n}=U^{n}+\alpha_{u}\left(U^{*}-U^{n}\right)$.

Note, for convenience we may interpret the relaxation parameter $r_{U}$ of the text via $\alpha_{U}=\left(1-r_{U}\right)$. Taking advantage of stage-1b equation (Eq.(A.1)), and re-arranging Eq.(A.3), yields:

$A_{U}\left(\tilde{U}^{*}-U^{n}\right)=\alpha_{u} \Delta t b_{U}$

an under-relaxed representation for equation stage-1b, casting velocity solution relaxation onto the local time-step, via scaling with factor $\alpha_{U}$.

To enforce relaxation upon stage- 2 alone, we introduce a relaxed pressure solution-component:

$\widetilde{P}^{n+1}=\alpha_{P} P^{n+1}+\left(1-\alpha_{P}\right) P^{n}=P^{n}+\alpha_{P}\left(P^{n+1}-P^{n}\right)$.

Revisiting stage-2 equation (Eq.(A.2)) with relaxation, and taking into account $\operatorname{Eq(A.5),~we~may~gather~}$ a relaxed stage-2 equation:

$$
K\left(\widetilde{P}^{n+1}-P^{n}\right)=\frac{\alpha_{P}}{\theta \Delta t} L U^{*} .
$$

Thus, at this fractional-equation stage, relaxing stage 2 is equivalent to scaling the local pressure equation time-step by $1 / \alpha_{P}$.

Finally, one may appreciate that relaxing velocity alone (and not pressure), conveys some relaxation into the local time-step at stage-2, through $U^{*}$ in the rhs-vector. Reconsidering the relaxed nondivergence-free velocity $\left(\widetilde{U}^{*}\right)$ of $\operatorname{Eq}(\mathrm{A} .3)$ and applying the discrete matrix equivalent to the divergence operator, $L$, yields:

$$
L \tilde{U}^{*}=\left(1-\alpha_{u}\right) L U^{n}+\alpha_{u} L U^{*}
$$

which will impact upon the system through the stage-2 equation accordingly,

$$
K\left(P^{n+1}-P^{n}\right)=\frac{1}{\theta \Delta t} L \tilde{U}^{*}=\frac{\alpha_{U}}{\theta \Delta t} L U^{*}+\frac{\left(1-\alpha_{U}\right)}{\theta \Delta t} L U^{n} .
$$

Here, we may appeal to the continuity equation to neglect the term $L U^{n} \approx 0$ on the right-hand-side of Eq.(A.8), giving:

$$
K\left(P^{n+1}-P^{n}\right) \approx \frac{\alpha_{U}}{\theta \Delta t} L U^{*}
$$

Therefore, we appreciate that by relaxing velocity at stage-1, we impart scaling on local time-steps at both stage-1 and stage-2. Similar arguments hold for the compressible flow context. 


\section{References}

1. J. Keshtiban F. Belblidia and M. F. Webster, Second-order schemes for steady weakly-compressible liquid flows, submitted to J. Comp. Phys., (2003). Available as CSR4-2003.

2. J. Keshtiban, F. Belblidia and M. F. Webster, Unified computational schemes for incompressible and weakly-compressible flows, submitted to Int. J. Num. Meth. Fluids, (2003). Available as CSR5-2003.

3. F.P.T. Baaijens, Mixed finite element methods for viscoelastic flow analysis: a review, J. Non-Newtonian Fluid Mech., 79 (1998) 361-385.

4. M.J. Crochet, A.R. Davis and K. Walters, Numerical Simulation of Non-Newtonian Flow, Elsevier, Amsterdam, 1984.

5. R. Keunings, Simulation of viscoelastic flow, in: C.L. Tucker (Ed.), Computer Modeling for Polymer Processing, Hanser, Munich, (1989), 404-469.

6. K. Walters and M.F. Webster, The distinctive CFD challenges of computational rheology, special issue Int. J. Num. Meth. Fluids, (Keynote: ECCOMAS Swansea, 1-26, 2001).

7. T.N. Phillips and A.J. Williams, Viscoelastic flow through a planar contraction using a semi-Lagrangian finite volume method, J. Non-Newtonian Fluid Mech., 87 (1999) 214-246.

8. P.J. Oliveira, F.T. Pinho, G.A. Pinto, Numerical simulation of non-linear elastic flows with a general collocated finite volume method, J. Non-Newtonian Fluid Mech., 79 (1998) 1-43.

9. A. Baloch, P. Townsend and M.F. Webster, On the simulation of highly elastic complex flows, J. NonNewtonian Fluid Mech., 59 (2/3) (1995) 111-128.

10. H. Matallah, P. Townsend and M.F. Webster, Recovery and stress-splitting schemes for viscoelastic flows, J. Non-Newtonian Fluid Mech., 75 (1998) 139-166.

11. J.M. Marchal and M.J. Crochet, Hermitian finite elements for calculating viscoelastic flow, J. NonNewtonian Fluid Mech., 20 (1986) 187-207.

12. N. Fietier and M.O. Deville, Time-dependent algorithms for the simulation of viscoelastic flows with spectral element methods: applications and stability, J. Comp. Phys., 186 (2003) 93-121.

13. P. Wapperom and M.F. Webster, A second-order hybrid finite-element/volume method for viscoelastic flows, J. Non-Newtonian Fluid Mech., 79 (1998) 405-431.

14. M. Aboubacar, M.F. Webster, A cell-vertex finite volume/element method on triangles for abrupt contraction viscoelastic flows, J. Non-Newtonian Fluid Mech., 98 (2001) 83-106.

15. R. Guènette, M. Fortin, A new mixed finite element method for computing viscoelastic flows, J. NonNewtonian Fluid Mech., 60 (1995) 27-52.

16. G.C. Georgiou, The time-dependent compressible Poiseuille and extrudate-swell flows of a Carreau fluid with slip at the wall, J. Non-Newtonian Fluid Mech., 109 (2003) 93-114.

17. E.A. Brujan, A first-order model for bubble dynamics in a compressible viscoelastic liquid, J. NonNewtonian Fluid Mech., 84 (1999) 83-103.

18. K.E. Barrett and A.C. Gotts, Finite element analysis of a compressible dynamic viscoelastic sphere using FFT, Comput. Struct., 80 (2202) 1615-1625.

19. P.G. Tait, HMSO, London 2(4), (1888).

20. C. Hirsch, Numerical Computation of Internal and External Flows, Volume 2: Computational mthods for inviscid and viscous flows, Wiley publication, (1999).

21. B. Poling, J. Prausnitz and J. O'Connell, Properties of Gases and Liquids, McGraw-Hill, $5^{\text {th }}$ edition, (2000).

22. D. Ding, P. Townsend and M.F. Webster, The iterative solution of Taylor-Galerkin augmented mass matrix equations, Int. J. Num. Meth. Eng., 35 (1992) 241-253.

23. D.M. Hawken, H.R. Tamaddon-Jahromi, P. Townsend and M.F. Webster, A Taylor-Galerkin based algorithm for viscous incompressible flow, Int. J. Num. Meth. Fluids, 10 (1990) 327-351.

24. T.N. Phillips and A.J. Williams, Comparison of creeping and inertial flow of an Oldroyd-B fluid through planar and axisymmetric contractions, J. Non-Newtonian Fluid Mech., 108 (2002) 25-47.

25. N.D. Waters and M.J. King, Unsteady flow of an elastico-viscous liquid, Rheol. Acta, 9 (1970) 245-355.

26. A.M. Alves, P.J. Oliveira and F.T. Pinho, Benchmark solutions for the flow of Oldroyd-B and PTT fluids in planar contractions, J. Non-Newtonian Fluid Mech., 110 (2003) 45-75.

27. P. Nithiarasu and O.C. Zienkiewicz, On stabilization of the CBS algorithm: Internal and external time steps, Int. J. Num. Meth. Eng., 48 (2000) 875-880. 


\section{Table and Figure Legend}

Table I. Mesh characteristic parameters.

Figure 1. Contraction flow schema.

Figure 2. Mesh refinement around the contraction, M1-M3 (mesh characteristics in Table I).

Figure 3: $\quad$ Compressible flow error norm convergence history for velocity, $E^{\mathrm{T}}(U)$, pressure, $E^{\mathrm{T}}(p)$, and stress $\mathrm{E}^{\mathrm{T}}(\mathrm{T})$, mesh $\mathrm{M} 1$; We $=0.1$, W\&K, quiescent ic. Top: $m_{i t n}=5, \Delta t=10^{-4}, 5 \times 10^{-4}$, $10^{-2}$. Bottom: $\Delta t=10^{-3}, m_{\text {it }}=1,3,5$.

Figure 4: Incompressible flow error norm convergence history for velocity, $E^{\mathrm{T}}(\mathrm{U})$, pressure, $\mathrm{E}^{\mathrm{T}}(\mathrm{p})$, and stress $\mathrm{E}^{\mathrm{T}}(\mathrm{T})$, mesh $\mathrm{M} 2$; a): Top left: $W e=0.1$, W\&K, quiescent ic. Top right: $W e=1.0$ ic. from $W e=0.1$. Middle b): $W e=0.1$, from quiescent ic. Bottom c): output of b), introducing under-relaxation.

Figure 5: Incompressible flow error norm convergence history for velocity, $E^{\mathrm{T}}(\mathrm{U})$, pressure, $\mathrm{E}^{\mathrm{T}}(\mathrm{p})$, and stress $\mathrm{E}^{\mathrm{T}}(\mathrm{T})$, mesh $\mathrm{M} 2 ; W e=1.0, \mathrm{~W} \& \mathrm{~K}$, quiescent ic. $\left(\Delta t=10^{-3}, m_{i t n}=5\right)$, with relaxation parameters $\left(r_{U}, r_{\tau}, r_{P}\right)$ as a): $\left.(0.0,0.0,0.0), \mathrm{b}\right):(0.3,0.3,0.3)$, c): $(0.7,0.7,0.7)$, d): $(0.7,0.7,0.0)$, e): $(0.0,0.7,0.0)$ and f): $(0.7,0.0,0.0)$.

Figure 6: Incompressible flow error norm convergence history for velocity, $E^{\mathrm{T}}(\mathrm{U})$, pressure, $\mathrm{E}^{\mathrm{T}}(\mathrm{p})$, and stress $\mathrm{E}^{\mathrm{T}}(\mathrm{T})$, mesh $\mathrm{M} 2 ; W e=1.0, \mathrm{~W} \& \mathrm{~K}$, quiescent ic. $\left(\Delta \mathrm{t}=10^{-3}, \mathrm{~m}_{\mathrm{itn}}=5\right)$ with relaxation parameters $\left(r_{U}, r_{\tau}, r_{P}\right)=(0.7,0.0,0.0)$, a): relaxation at $1^{\text {st }}$ TGPC stage, $\left.\mathrm{b}\right)$ : relaxation at $3^{\text {rd }}$ TGPC stage.

Figure 7: $\quad$ Error norm convergence history for velocity, $E^{\mathrm{T}}(\mathrm{U})$, pressure, $\mathrm{E}^{\mathrm{T}}(\mathrm{p})$, and stress $\mathrm{E}^{\mathrm{T}}(\mathrm{T})$, for different meshes (M1: top, M2: middle, M3: bottom) and different flow scenarios (left: incompressible, centre: weakly-compressible with $(m, B)=\left(10^{4}, 10^{4}\right)$, right: compressible with $(m, B)=\left(4,10^{2}\right)$. We $=1.0, R e=1.0$.

Figure 8: $\quad$ Pressure $P$ contours, different meshes (M1: top, M2: centre, M3: bottom), left: incompressible, middle: weakly-compressible and right: compressible flow. $W e=1.0$, $R e=1.0$.

Figure 9: $\quad$ Principal stress $\mathrm{N}_{1}$ contours, different meshes (M1: top, M2: centre, M3: bottom), left: incompressible, middle: weakly-compressible and right: compressible flow. $W e=1.0$, $R e=1.0$.

Figure 10: $\quad$ Stress $\tau_{x y}$ contours, different meshes (M1: top, M2: centre, M3: bottom), left: incompressible, middle: weakly-compressible and right: compressible flow. $W e=1.0$, $R e=1.0$.

Figure 11: $\quad$ Streamline contours, incompressible (left) and compressible flow (right), mesh M3. $W e=1.0, R e=1.0$.

Figure 12: Density (left) and Mach number (right) contours for compressible flow setting, mesh M3. $W e=1.0, R e=1.0$.

Figure 13: $\quad$ Stress profiles ( $\tau_{x x}$ : top, $\tau_{x y}$ : middle, $\tau_{y y}$ : bottom) along $y=0.3$, different meshes (M1, M2, M3) and flow scenarios (incompressible: left, weakly-compressible: centre, compressible: right). $W e=1.0, R e=1.0$.

Figure 14a: Profile sampling $x$-positions for principal stress $\mathrm{N}_{1}$ along downstream channel.

Figure 14b-d: Principal stress $\mathrm{N}_{1}$-profiles across downstream channel section, sampling $x$-positions, mesh M3, b: incompressible, c: weakly-compressible, d: compressible. $W e=1.0$, $R e=1.0$.

Figure 15: $\quad$ Principal stress $\mathrm{N}_{1}$-profiles, sampling $x$-positions near contraction zone, mesh $\mathrm{M} 3$, a: incompressible, b: weakly-compressible, c: compressible. $W e=1.0, R e=1.0$.

Figure 16: $\quad$ Stress profiles: $\tau_{x x}$ (right) and $\tau_{x y}$ (left) along downstream channel. Boundary layer growth for incompressible, weakly-compressible and compressible flow conditions, mesh M3. $W e=1.0, R e=1.0$ (scaling indicated). 
Table I. Mesh characteristic parameters.

\begin{tabular}{|l|c|c|c|}
\cline { 2 - 4 } \multicolumn{1}{c|}{} & M1 & M2 & M3 \\
\hline Elements & 980 & 1140 & 2987 \\
\hline Nodes & 2105 & 2427 & 6220 \\
\hline Vertex Nodes & 563 & 644 & 1617 \\
\hline d.o.f. & 8983 & 9708 & 14057 \\
\hline Corner mesh density & 28 & 63 & 201 \\
\hline $\mathrm{R}_{\min }$ & 0.024 & 0.023 & 0.006 \\
\hline $\mathrm{R}_{\max }$ & 0.126 & 0.131 & 0.095 \\
\hline
\end{tabular}

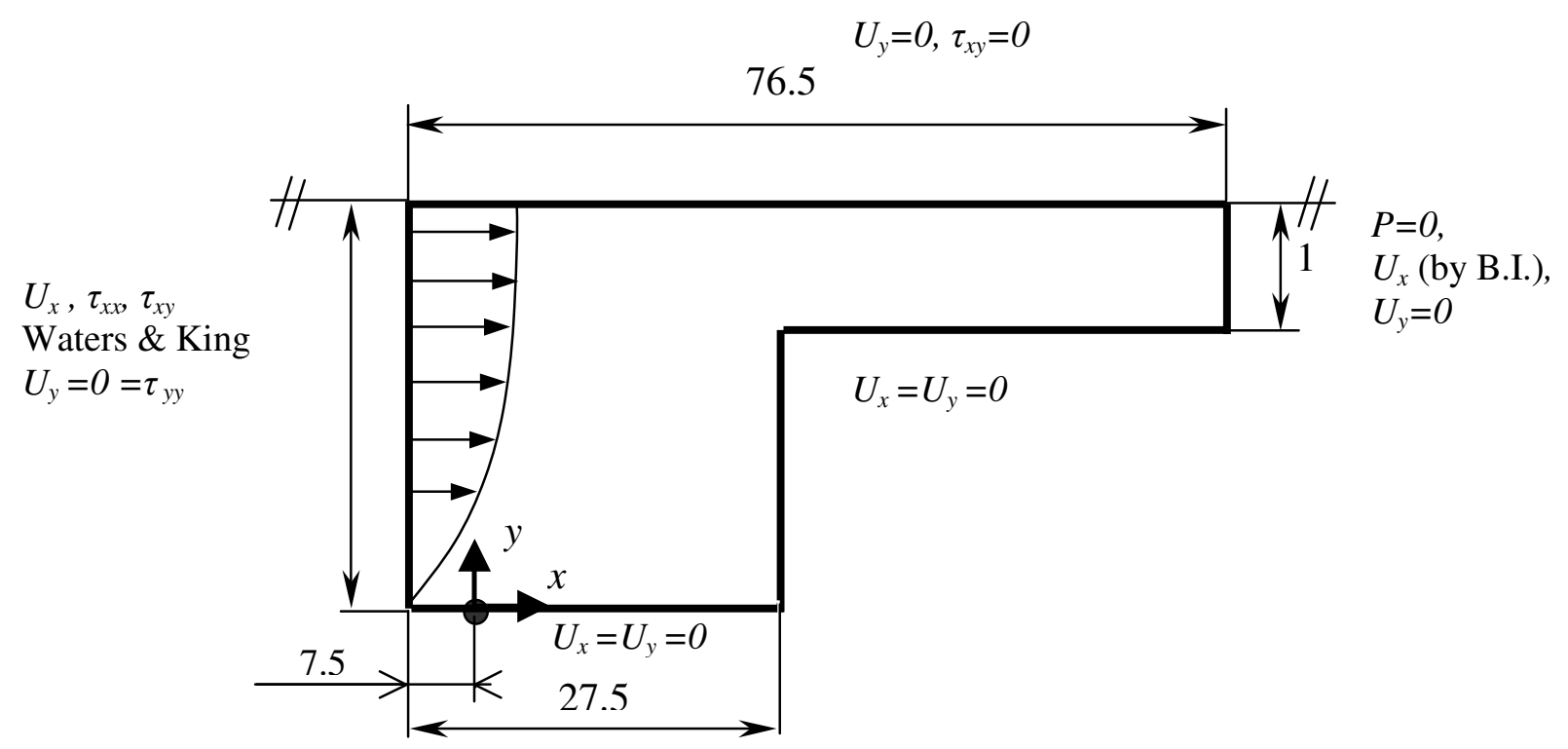

Figure 1. Contraction flow schema. 

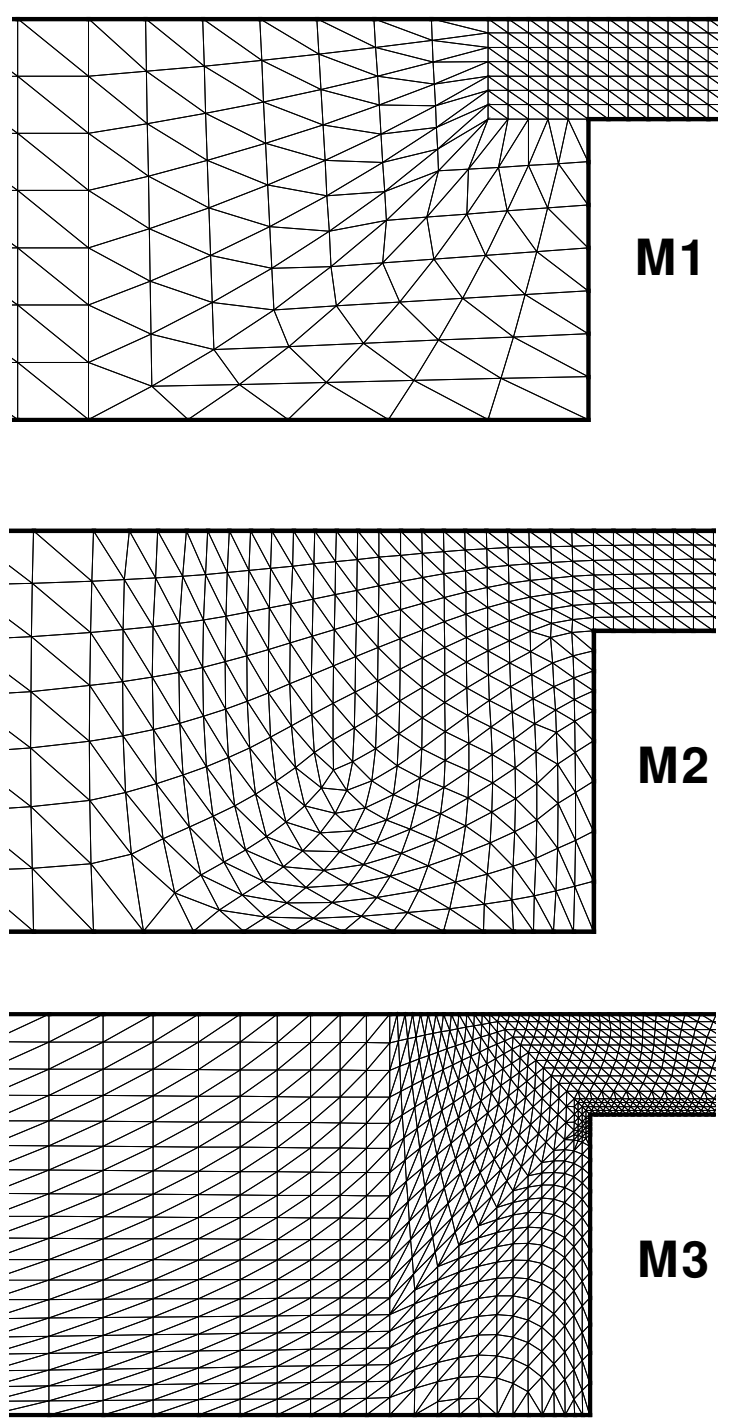

Figure 2. Mesh refinement around the contraction, M1-M3 (mesh characteristics in Table I). 

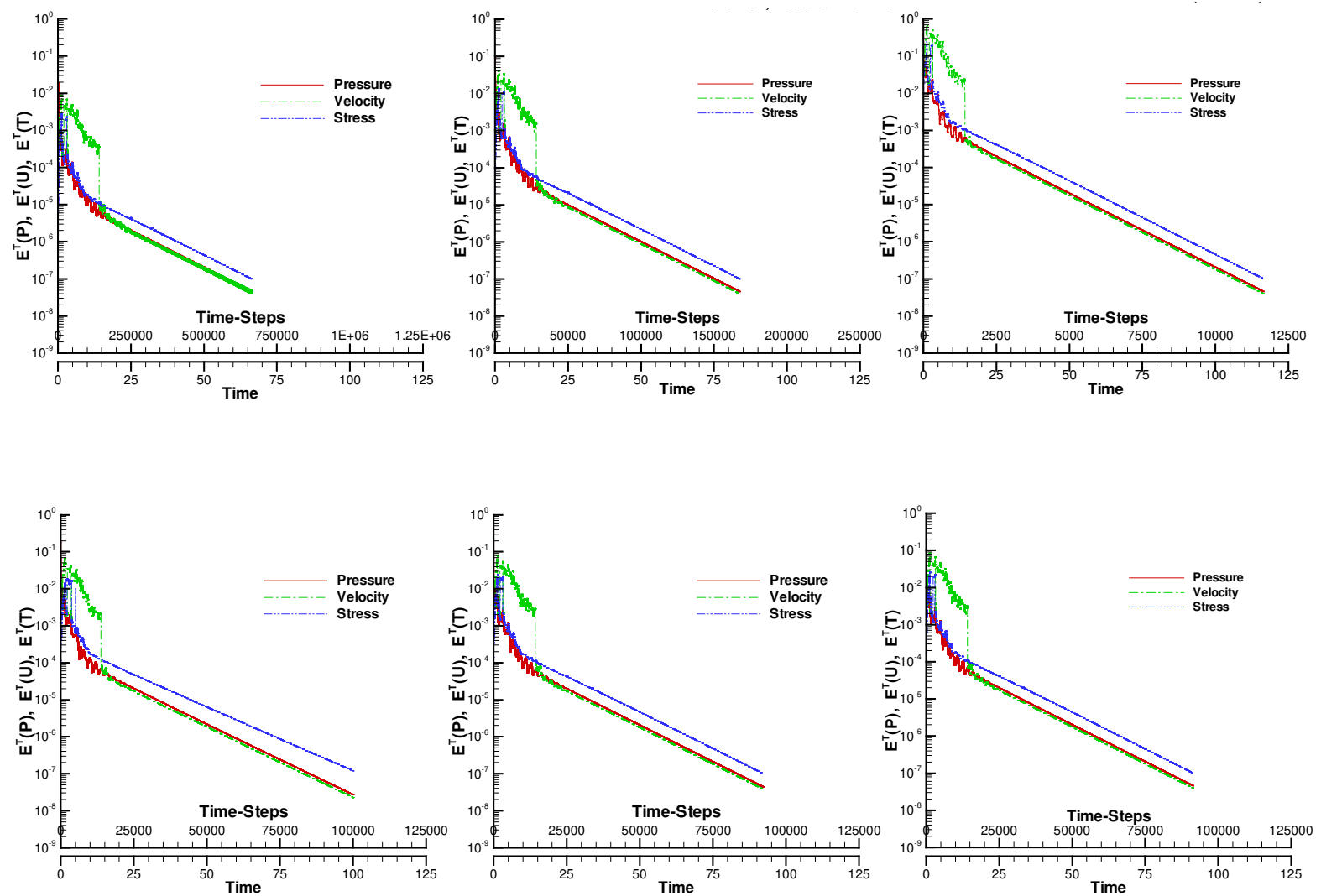

Figure 3: Compressible flow error norm convergence history for velocity, $E^{\mathrm{T}}(\mathrm{U})$, pressure, $\mathrm{E}^{\mathrm{T}}(\mathrm{p})$, and stress $\mathrm{E}^{\mathrm{T}}(\mathrm{T})$, mesh $\mathrm{M} 1 ; W e=0.1$, W\&K, quiescent ic. Top: $m_{i t n}=5, \Delta t=10^{-4}, 5 \times 10^{-4}, 10^{-2}$. Bottom: $\Delta t=10^{-3}, m_{i t n}=1,3,5$. 
a)
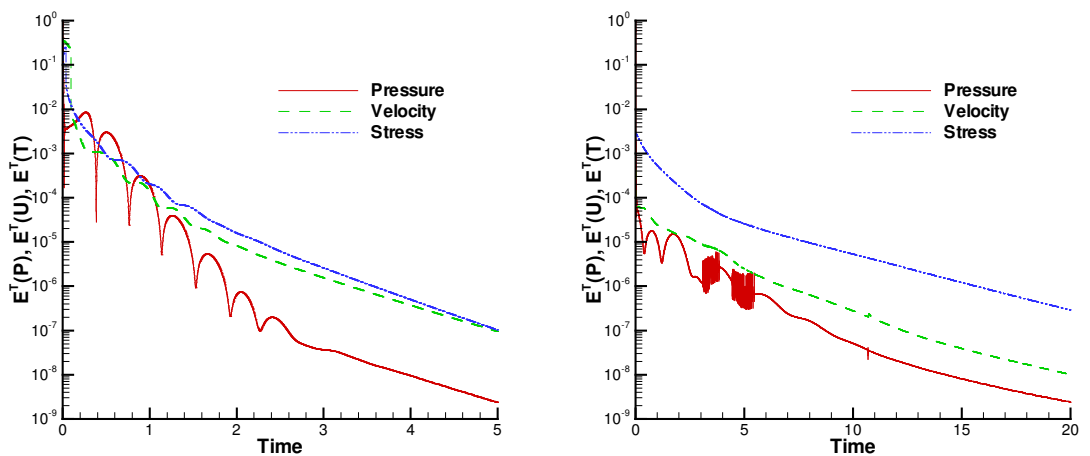

b)

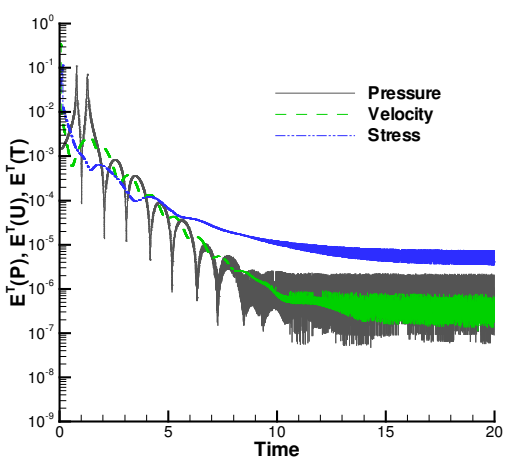

c)

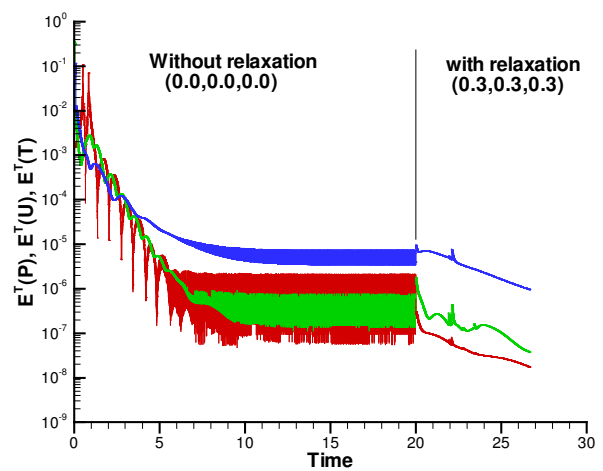

Figure 4: Incompressible flow error norm convergence history for velocity, $E^{\mathrm{T}}(U)$, pressure, $E^{\mathrm{T}}(p)$, and stress $\mathrm{E}^{\mathrm{T}}(\mathrm{T})$, mesh $\mathrm{M} 2$; a): Top left: $W e=0.1$, W\&K, quiescent ic. Top right: $W e=1.0$ ic. from $W e=0.1$. Middle $\mathrm{b}$ ): $W e=0.1$, from quiescent ic. Bottom c): output of $\mathrm{b}$ ), introducing under-relaxation. 


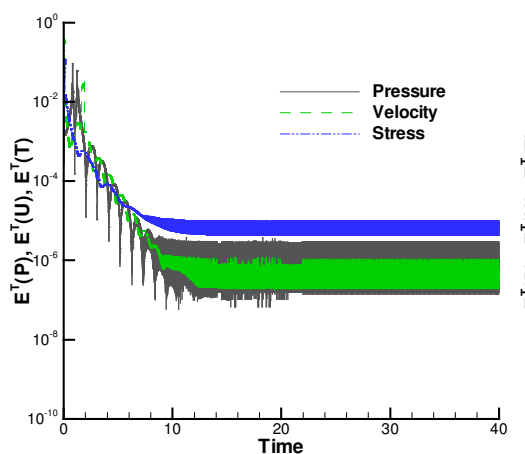

a)

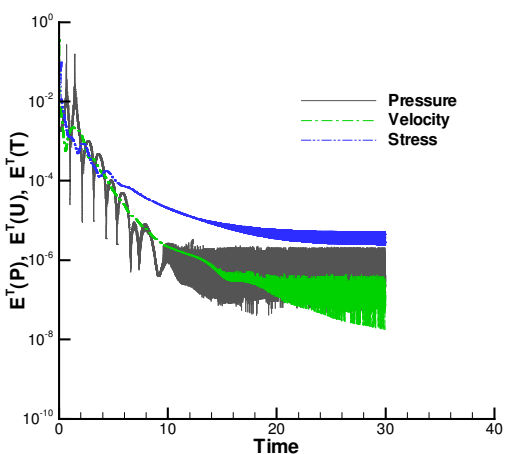

b)

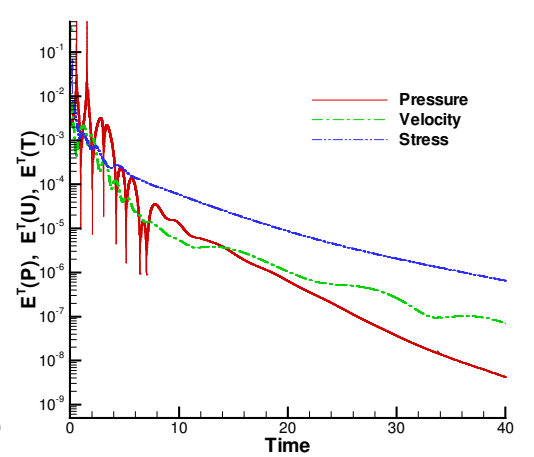

c)

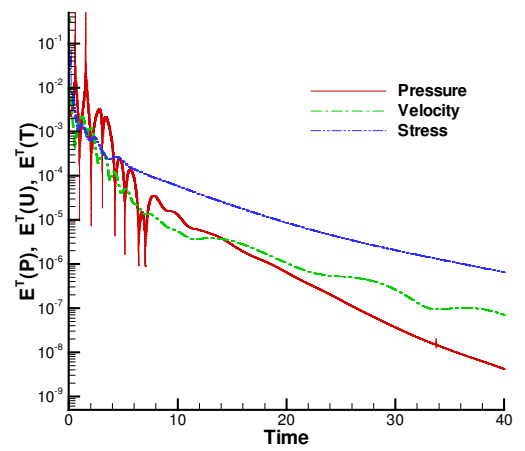

d)

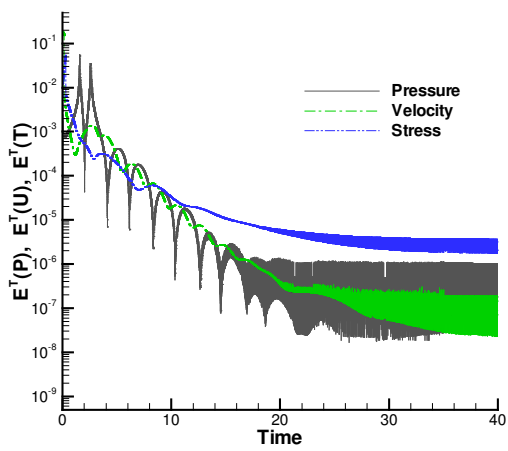

e)

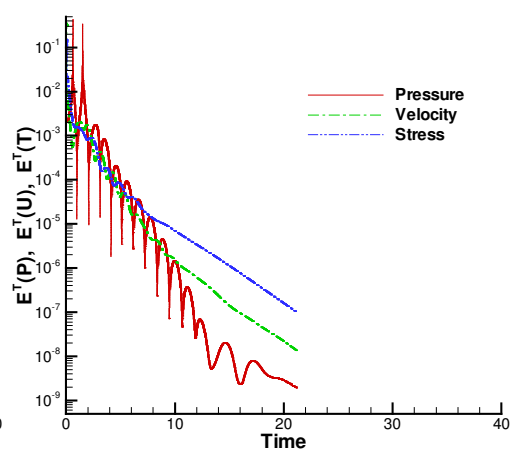

f)

Figure 5: Incompressible flow error norm convergence history for velocity, $E^{\mathrm{T}}(\mathrm{U})$, pressure, $\mathrm{E}^{\mathrm{T}}(\mathrm{p})$, and stress $\mathrm{E}^{\mathrm{T}}(\mathrm{T})$, mesh $\mathrm{M} 2 ; W e=1.0, \mathrm{~W} \& \mathrm{~K}$, quiescent ic., $\left(\Delta t=10^{-3}, m_{i t n}=5\right)$ with relaxation parameters $\left(r_{U}, r_{\tau}, r_{P}\right)$ as a): $\left.\left.\left.(0.0,0.0,0.0), \mathrm{b}\right):(0.3,0.3,0.3), \mathrm{c}\right):(0.7,0.7,0.7), \mathrm{d}\right):(0.7,0.7,0.0)$, e): $(0.0,0.7,0.0)$ and $f):(0.7,0.0,0.0)$. 


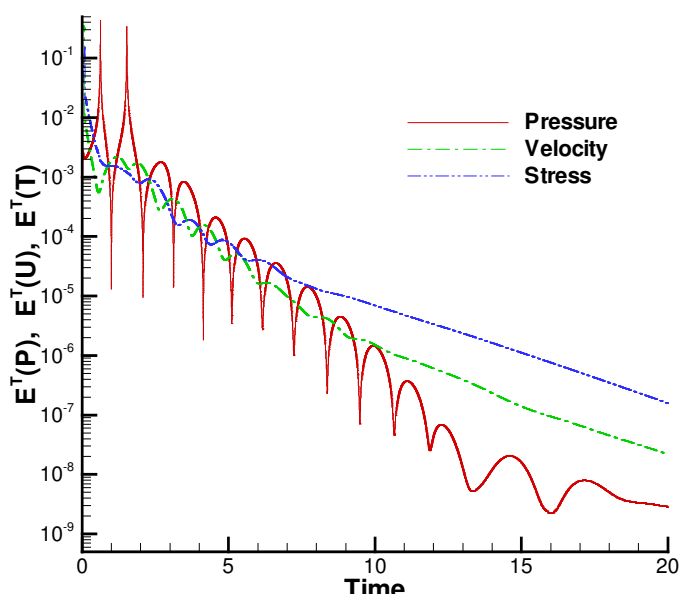

a)

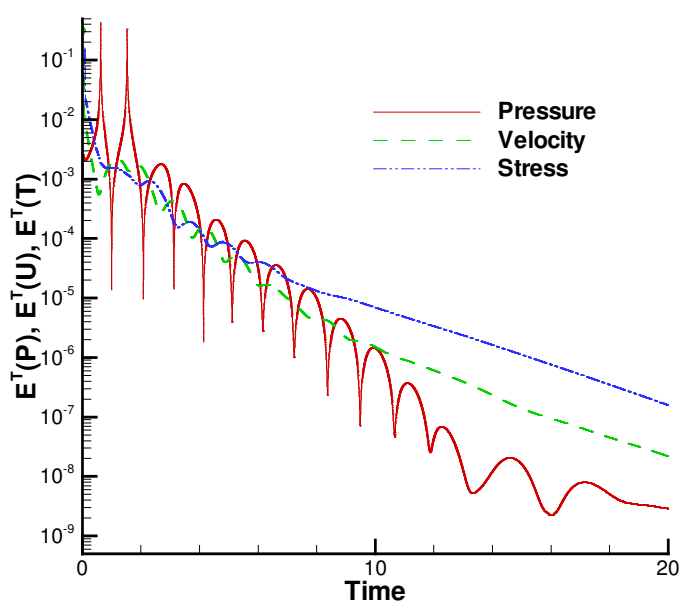

b)

Figure 6: Incompressible flow error norm convergence history for velocity, $E^{\mathrm{T}}(U)$, pressure, $E^{\mathrm{T}}(p)$, and stress $\mathrm{E}^{\mathrm{T}}(\mathrm{T})$, mesh $\mathrm{M} 2 ; W e=1.0, \mathrm{~W} \& \mathrm{~K}$, quiescent ic. $\left(\Delta \mathrm{t}=10^{-3}, \mathrm{~m}_{\mathrm{itn}}=5\right)$ with relaxation parameters $\left(r_{U}, r_{v}, r_{P}\right)=(0.7,0.0,0.0)$, a): relaxation at $1^{\text {st }}$ TGPC stage, b): relaxation at $3^{\text {rd }}$ TGPC stage. 
Incompressible

Weakly-compressible

Compressible

$(m, B)=\left(10^{4}, 10^{4}\right)$

$(m, B)=\left(4,10^{2}\right)$
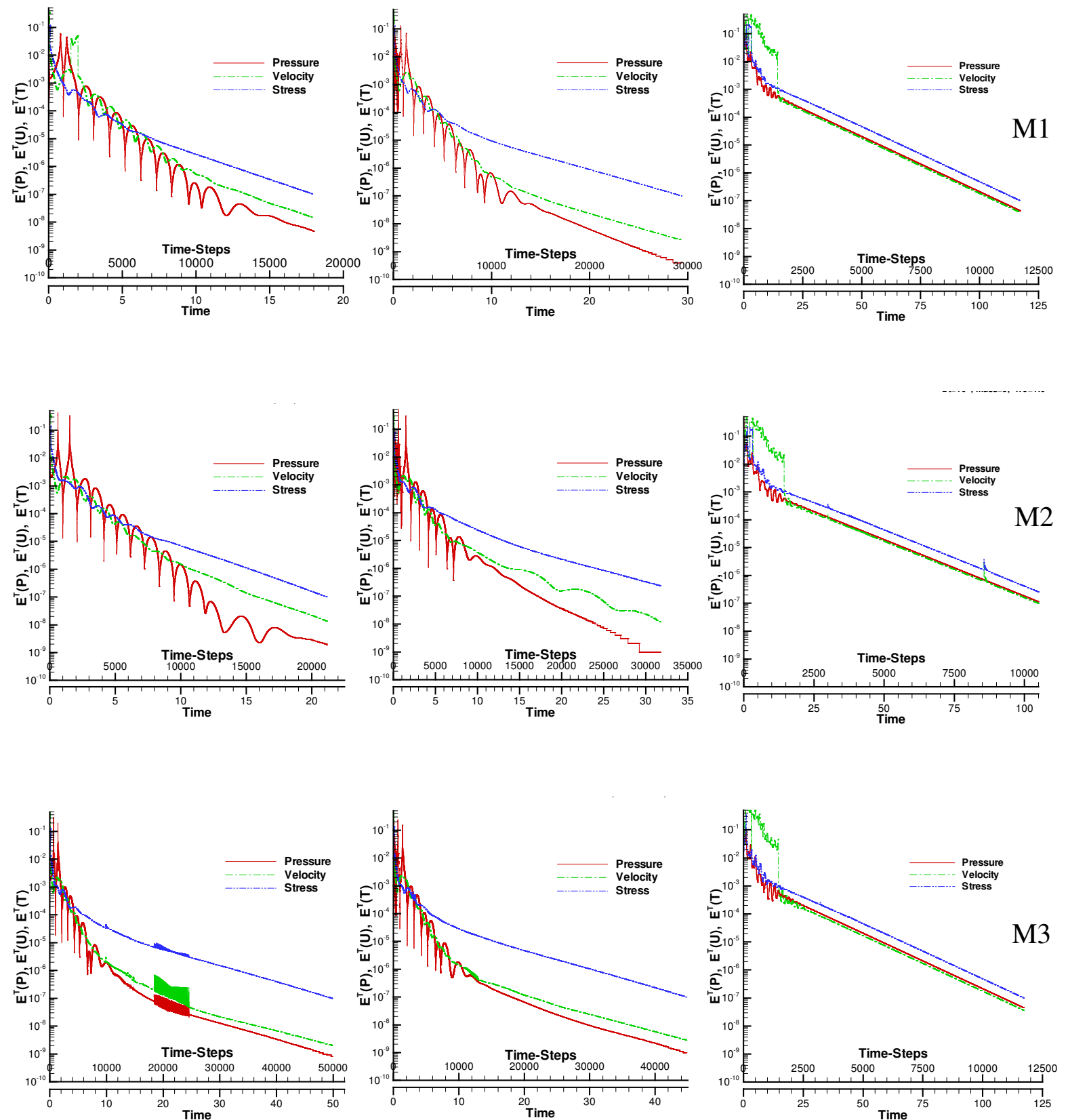

Figure 7: Error norm convergence history for velocity, $E^{T}(U)$, pressure, $E^{T}(p)$, and stress $E^{T}(T)$, for different meshes (M1: top, M2: middle, M3: bottom) and different flow scenarios (left: incompressible, centre: weakly-compressible with $(m, B)=\left(10^{4}, 10^{4}\right)$, right: compressible with $(m, B)=\left(4,10^{2}\right) . W e=1.0, R e=1.0$. 


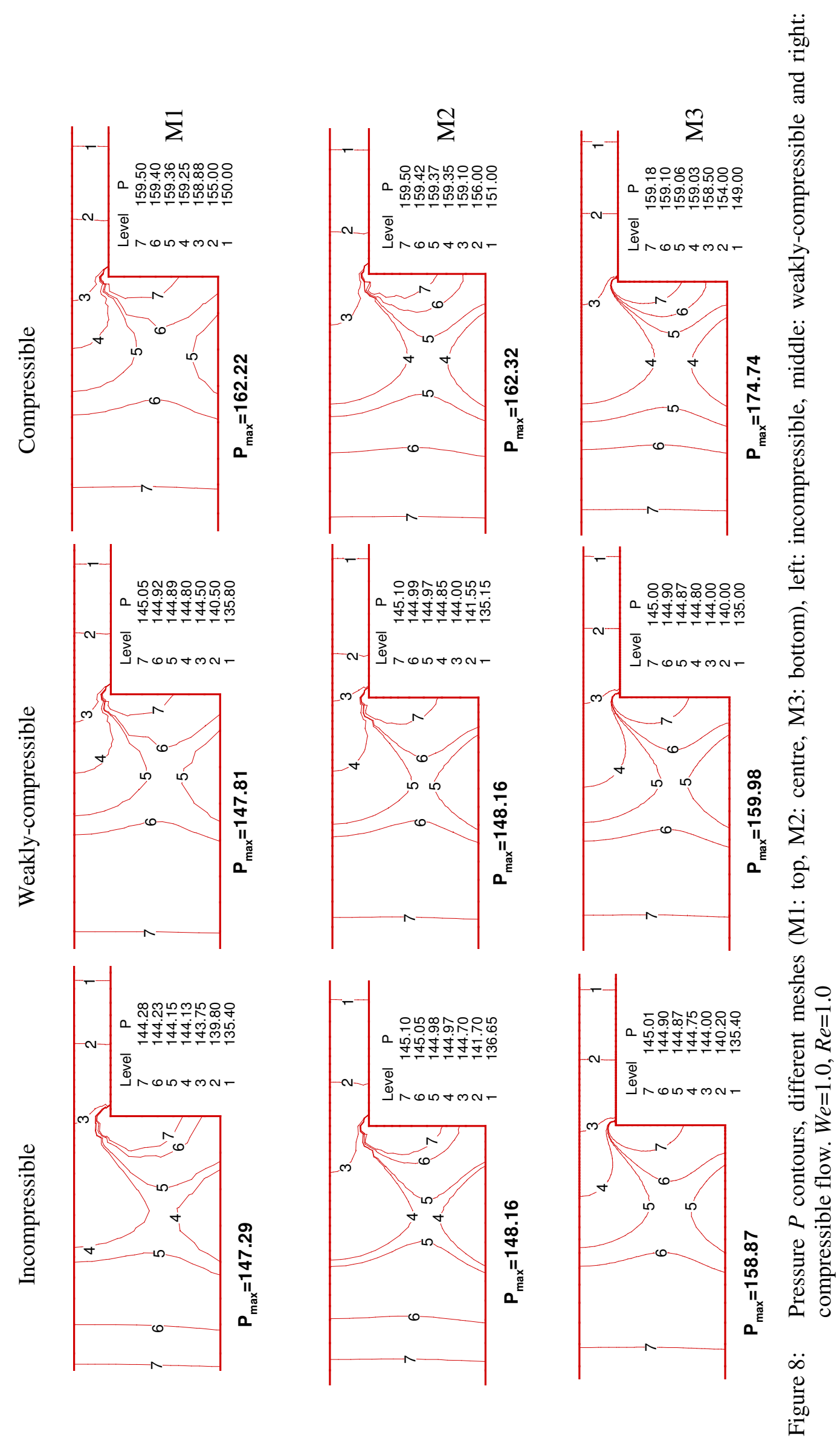



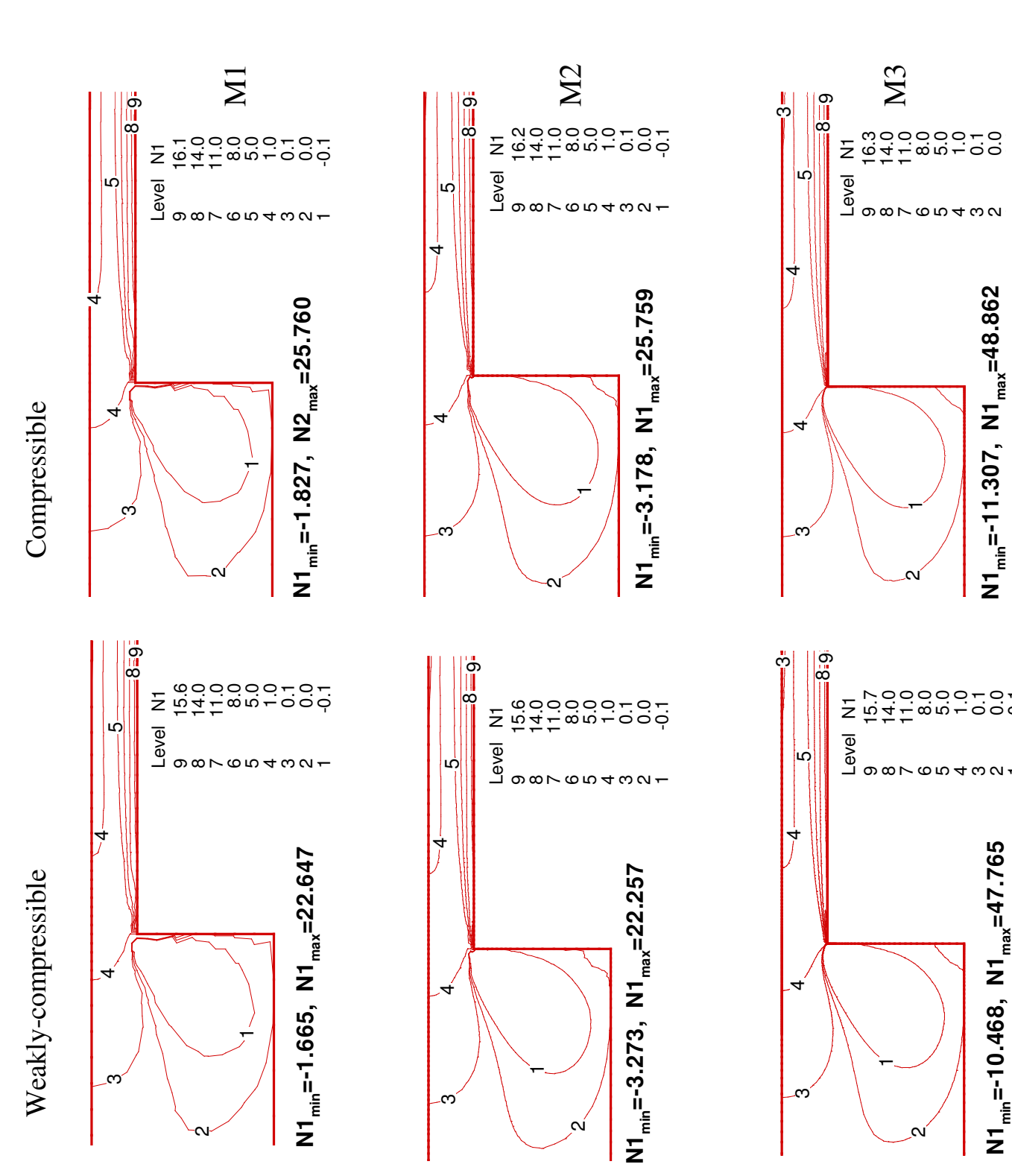

로

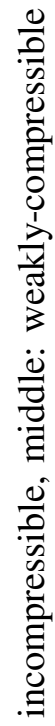

苞

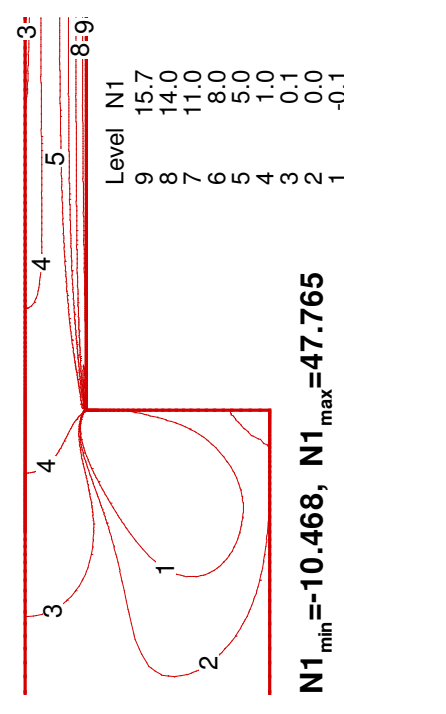

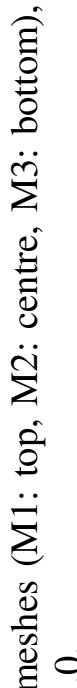
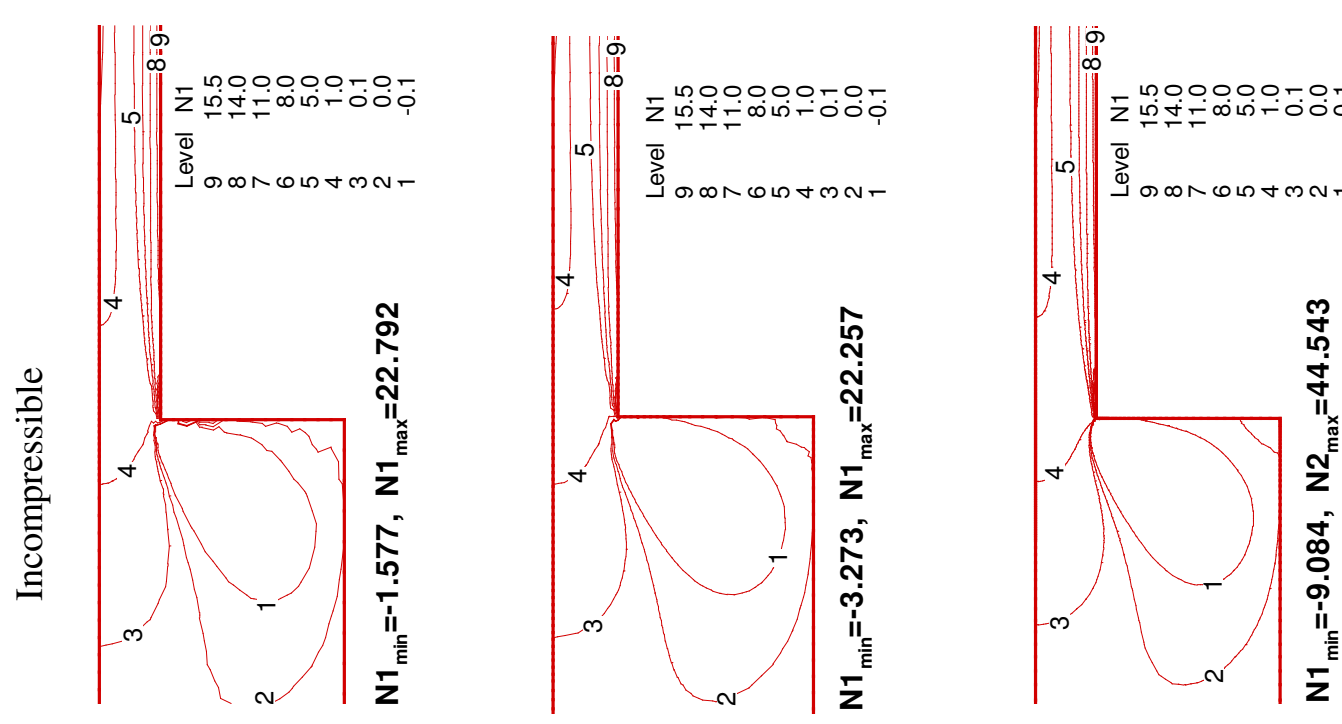

चू $\stackrel{0}{=0}$ ڤิ 응 乙告 ป1 के พ พ . 苛

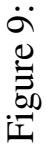



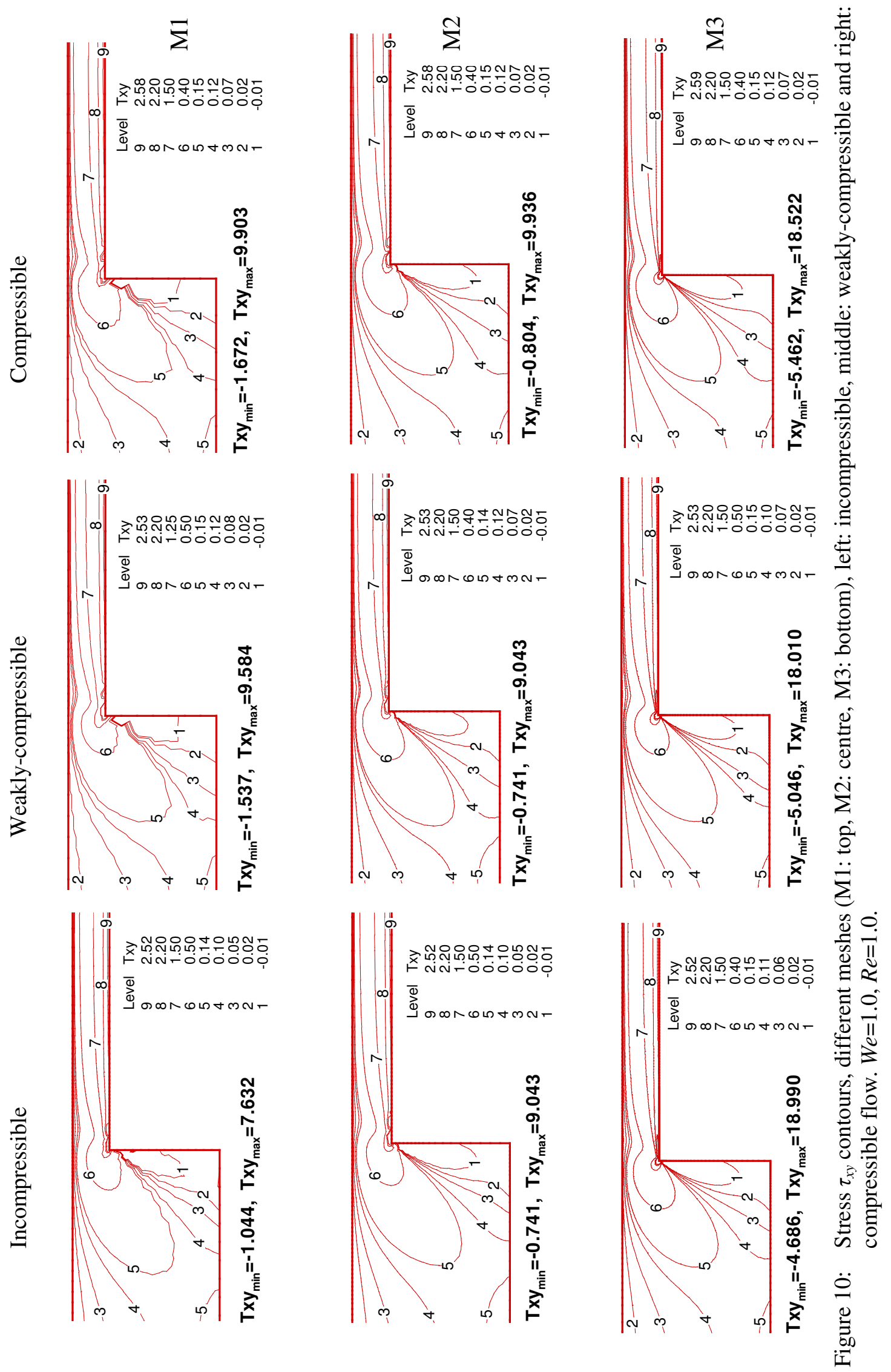

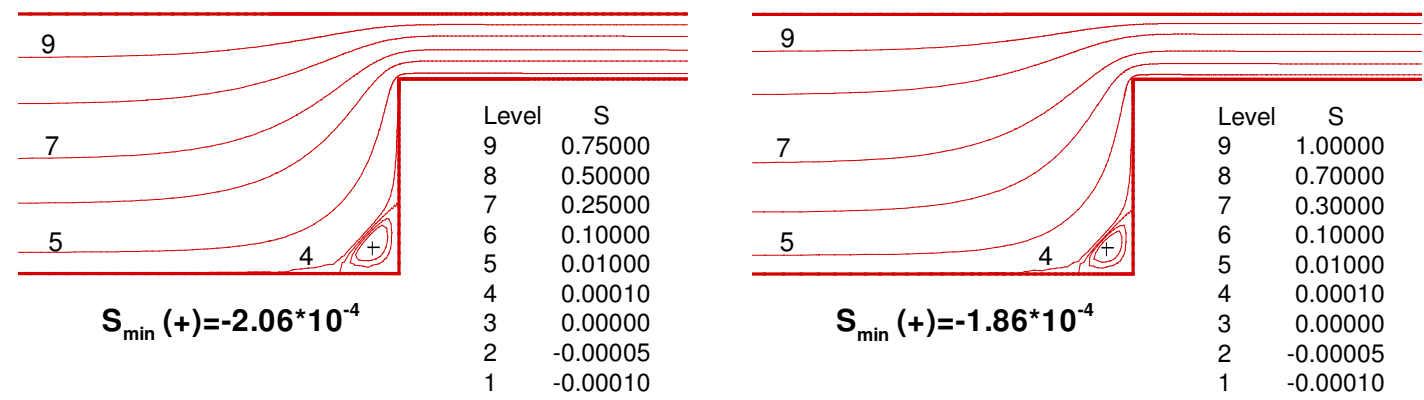

Figure 11: Streamline contours, incompressible (left) and compressible flow (right), mesh M3. We=1.0, $\operatorname{Re}=1.0$.
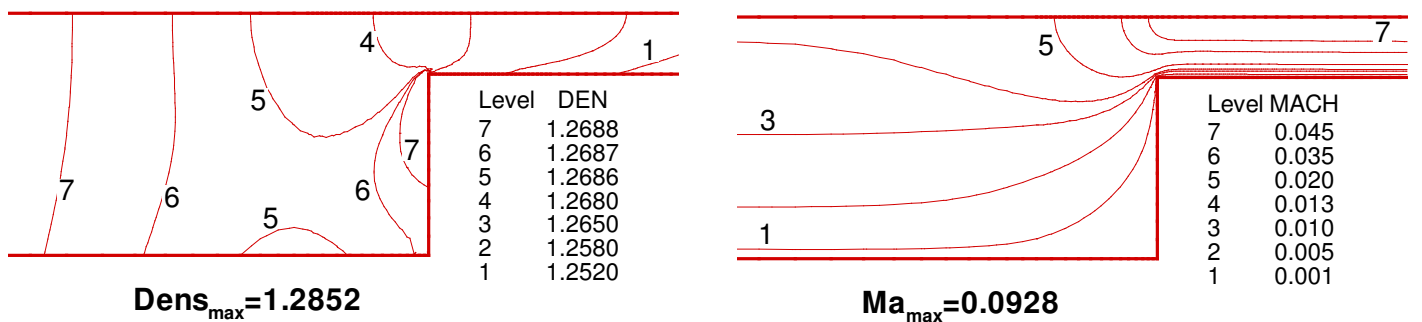

Figure 12: Density (left) and Mach number (right) contours for compressible flow setting, mesh M3. $W e=1.0, R e=1.0$. 
Incompressible
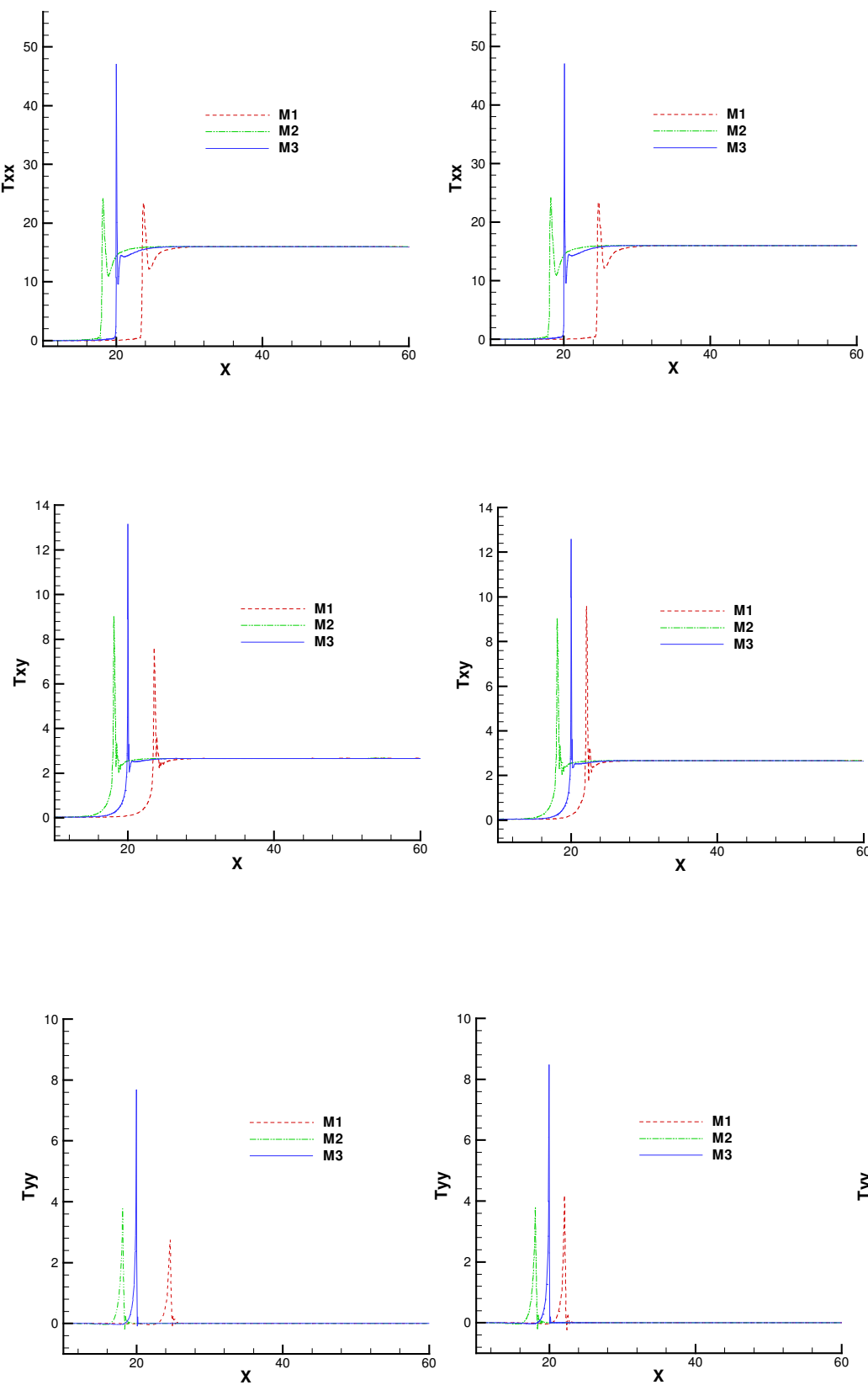

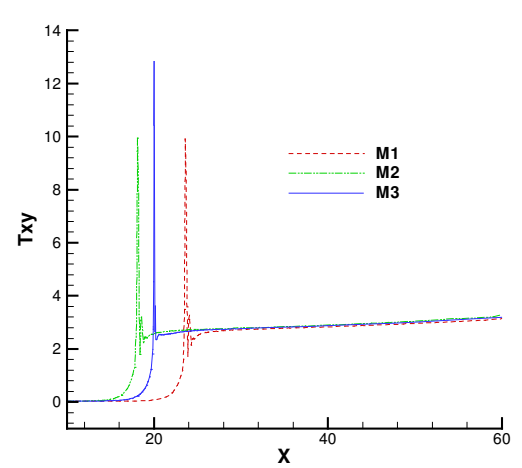

Compressible

$(m, B)=\left(4,10^{2}\right)$
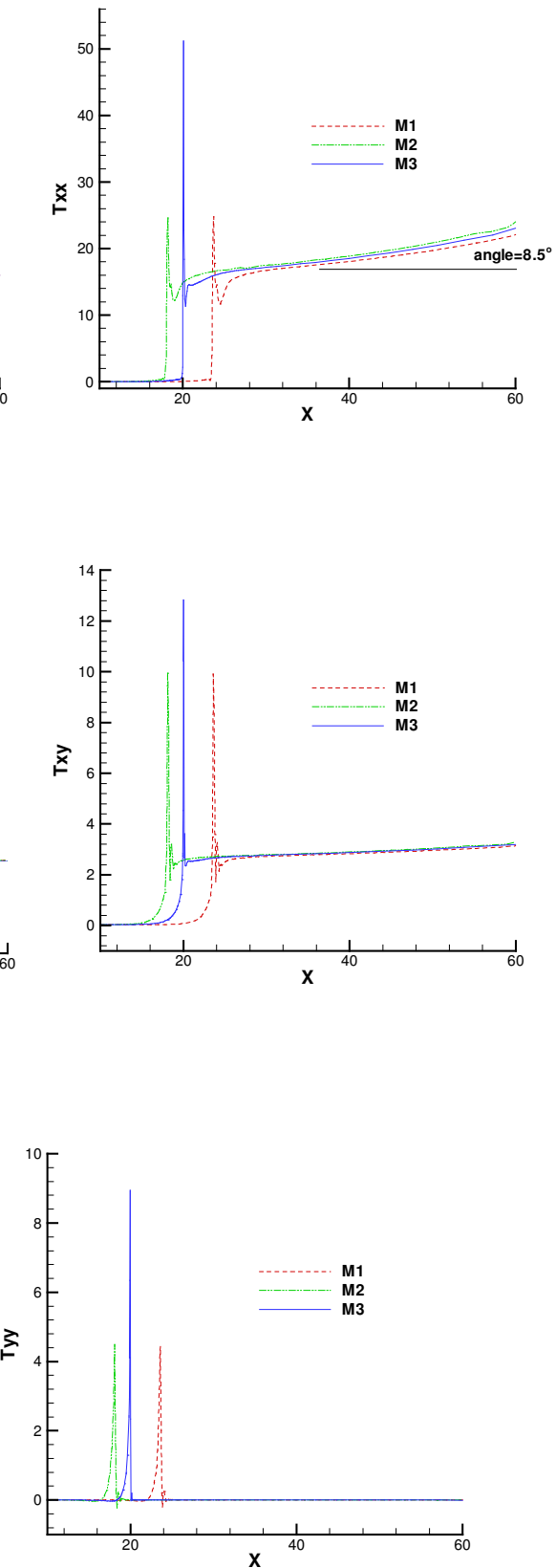

Figure 13: Stress profiles ( $\tau_{x x}$ : top, $\tau_{x y}$ : middle, $\tau_{y y}$ : bottom) along $y=0.3$, different meshes (M1, M2, M3) and flow scenarios (incompressible: left, weakly-compressible: centre, compressible: right). $W e=1.0, R e=1.0$. 


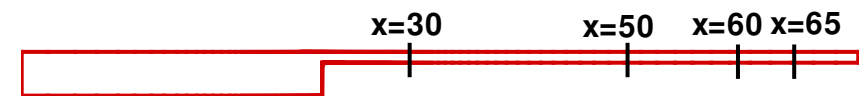

Figure 14a: Profile sampling $x$-positions for principal stress $\mathrm{N}_{1}$ along downstream channel.

Incompressible

$$
\begin{gathered}
\text { Weakly-compressible } \\
(m, B)=\left(10^{4}, 10^{4}\right)
\end{gathered}
$$

Compressible

$(m, B)=\left(4,10^{2}\right)$

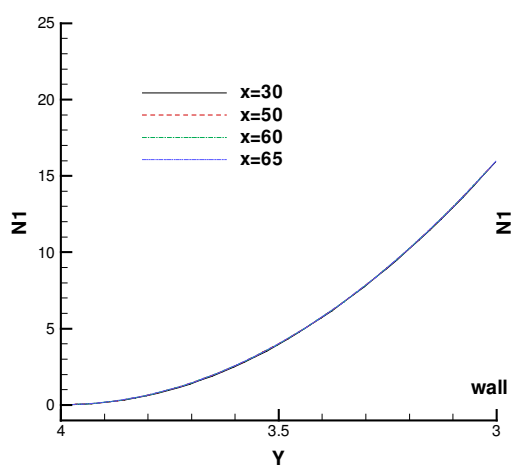

b)

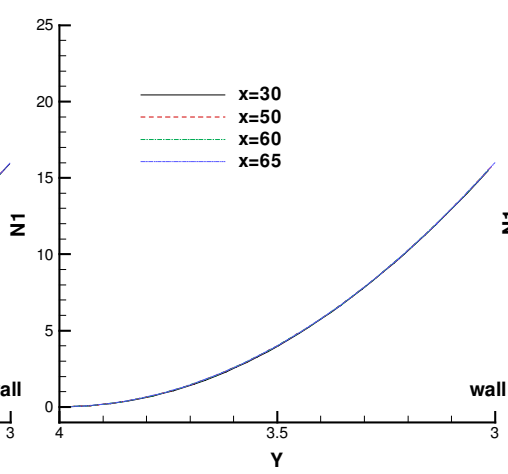

c)

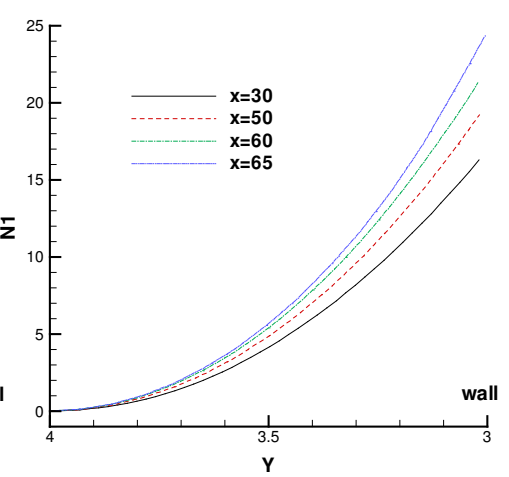

d)

Figure 14b-d: Principal stress $\mathrm{N}_{1}$-profiles across downstream channel section, sampling $x$-positions, mesh M3, b: incompressible, c: weakly-compressible, d: compressible. $W e=1.0, R e=1.0$. 

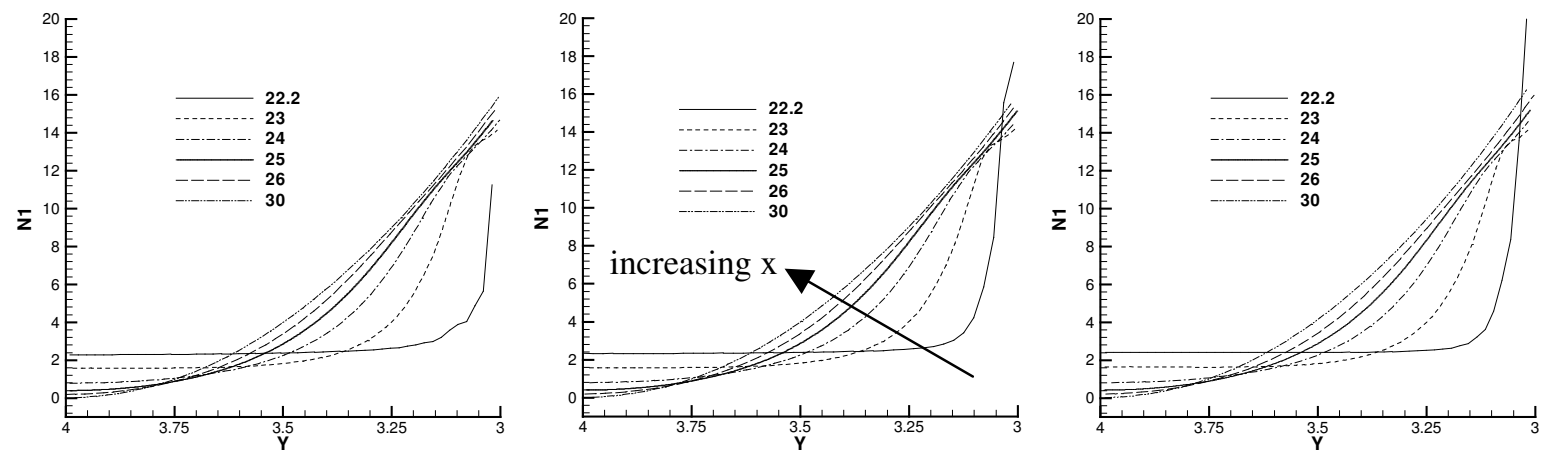

Figure 15: Principal stress $\mathrm{N}_{1}$-profiles, sampling $x$-positions near contraction zone, mesh $\mathrm{M} 3$, a: incompressible, b: weakly-compressible, c: compressible. $W e=1.0, R e=1.0$.
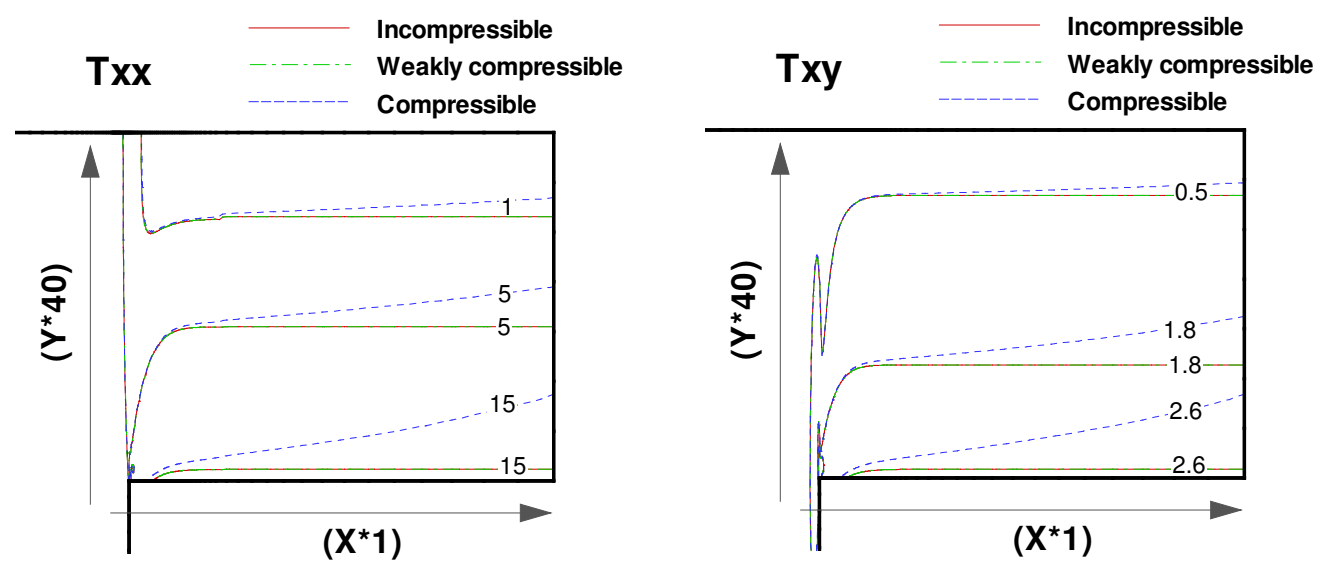

Figure 16: Stress profiles: $\tau_{x x}$ (right) and $\tau_{x y}$ (left) along downstream channel. Boundary layer growth for incompressible, weakly-compressible and compressible flow conditions, mesh M3. We $=1.0$, $R e=1.0$ (scaling indicated). 